\title{
Stochastic Versions of Turnpike Theorems in the Sense of Uniform Topology
}

\author{
Darong Dai* \\ School of Business, Nanjing University, Nanjing 210093, P. R. China \\ E-mail: daidarong998@163.com
}

\begin{abstract}
In this paper, a stochastic endogenous aggregative growth model is constructed and two main results are established, based on endogenous horizon of the economy and endogenous terminal capital stock, which is also efficient capital accumulation in some sense. First, strong turnpike theorems under uncertainty and in the sense of uniform topology are obtained; second, inefficacy of temporary fiscal policy, which is chosen to be capital income taxation, has been demonstrated in comparatively weak conditions different from Yano (1998)'s.
\end{abstract}

Key Words: Stochastic endogenous growth; Uniform-topology turnpike theorems; Endogenous terminal stock; Endogenous welfare function; Capital income taxation.

JEL Classification Numbers: C61, C62, E21, E22, E62.

\section{INTRODUCTION}

Our goal of this paper is to study turnpike theorems and the effects of temporary fiscal policy, which is specifically chosen to be capital income taxation, in a stochastic endogenous growth model, with the source of uncertainty is the population size of the representative household. Competitive equilibrium assumptions are also employed, that is the firm, using AK production technology (e.g., Barro, 1990; Rebelo, 1991; Turnovsky, 2000; Aghion, 2004), has zero profit in the equilibrium of the economy.

In the past several decades, the so-called turnpike theorems have been extensively studied and well understood. Most of them (e.g., Morishima, 1961, 1965; Tsukui, 1966, 1967; McKenzie, 1963, 1976; Winter, 1967; Coles, 1985; Yano, 1984a, 1984b, 1985; Bewley, 1982; Gale, 1967; Gantz, 1980;

\footnotetext{
* Corresponding author.
} 
Drandakis, 1966; and Araujo and Scheinkman, 1977), however, focused on the following four types of specifications: first, multi-sector economies or general equilibrium models with many consumers and producers; second, fiscal policies are generally excluded in their models; third, they just concern the deterministic cases, i.e., uncertainty is usually excluded in their models; and fourth, the horizon of the abstract economy, fixed finite or infinite, and the terminal stock are all exogenously given. There are certainly some exceptions, for instance, fiscal policy has been considered and carefully studied in Yano (1998)'s model. Rather, Yano demonstrated that a temporary change in fiscal policy has almost no effect on present and future consumption with taking the general equilibrium price effect into account in a dynamic general equilibrium model, hinging on the following three types of assumptions: first, the existence of an interior dynamic general equilibrium; second, the smoothness of utility and production functions; and third, the uniqueness of a stationary equilibrium consumption vector in the case of undiscounted future utilities. Moreover, Joshi (1997) provided a comprehensive development of turnpike theory in a stochastic aggregative growth model, extending the classical turnpike theory to general non-convex and non-stationary environments. Although the model in the paper is a stochastic aggregative growth model with the effect of temporary fiscal policy being thoroughly examined, unlike Yano (1998), our conclusion of the inefficacy of temporary fiscal policy on equilibrium consumption path holds true in comparatively weak conditions, say, given the initial level of capital stock sufficiently low, in the case of discounted future utilities, and in a more realistic stochastic environment. What's more, here the source of uncertainty is supposed to be population size of the representative household, thereby leading to a stochastic diffusion process of capital accumulation, while Joshi (1997) directly and exogenously introducing the stochastic environments as independent variables into production functions.

Furthermore, when discussing efficient capital accumulation (e.g., Gong and Zou, 2000, 2002), efficiency is usually defined with reference to the final state (see, Radner, 1961; Kurz, 1965) or the terminal stocks (see, McKenzie, 1963, 1976). In this paper, also, the terminal stock, equivalent to efficient capital accumulation in some sense, is endogenously determined as well as the stopping time of the economy, which is an optimal stopping time that maximizes the final-state objective function of the representative household, i.e., choosing a minimum time so as to maximize the discounted utility function, which, to some extent, resembles Kurz (1965)'s specification, that is, minimizing the time to economic maturity. And hence it is argued that one contribution of this paper is to show that the horizon of the economy and the terminal capital stock, also efficient capital accumulation, can be simultaneously and endogenously determined, thereby endogenous- 
ly generating a single welfare function in an aggregated model of optimal growth. And it is easy to see that our result is a natural correspondence to Bewley (1982)'s, which shows that the social welfare function is endogenously determined by the market mechanism in decentralized models of optimal growth.

Finally, noting that existing turnpike theorems, in optimal growth theory, as Yano (1985) argued that, can be summarized as the following two types, one is neighborhood turnpike theorem (see, Yano, 1984b; McKenzie, 1982 ) which asserts that an optimal path in a growth model converges to a small neighborhood of a stationary path, the other is asymptotic turnpike theorem (e.g., Araujo and Scheinkman, 1977; Bewley, 1982; Yano, 1985) which means that an optimal path converges to a stationary path. Here, we have proved much stronger turnpike theorems in the sense of uniform topology, which we may call uniform-topology turnpike theorems, and this would appear to be the second innovation of the present paper.

The rest of the paper is organized as follows: section 2 presents the model and our key theorems, section 3 gives some concluding remarks and the appendix provides the main mathematical derivations.

\section{THE MODEL}

We assume that the economy admits a representative household with instantaneous utility function $u(\cdot)=\ln (\cdot)$, i.e., with log preferences. Our goal in the paper is to investigate turnpike theorems in a stochastic abstract economy, and here the source of uncertainty is the population size $L(t)$ (e.g., Merton, 1975), which grows in accordance with the following stochastic differential equation (SDE),

$$
d L(t)=n L(t) d t+\sigma L(t) d B(t)
$$

where $\sigma \in \mathbb{R}$ is some constant and $B(t)$ is a standard Brownian motion on a given complete probability space $\left(\Omega, \mathcal{F},\left\{\mathcal{F}_{t}\right\}_{t \geq 0}, \mathbb{P}\right)$ with natural filtration $\left\{\mathcal{F}_{t}\right\}_{t \geq 0}$ and $B(0)=0 \mathbb{P}$-a.s..

To prepare for the household optimization, let us denote the asset holdings of the representative household at time $t$ by $\mathbb{A}(t)$, then we get the following law of motion for the total assets of the household

$$
\dot{\mathbb{A}}(t)=\left(1-r^{t}\right) r(t) \mathbb{A}(t)+w(t) L(t)-c(t) L(t)
$$

where $c(t)$ is consumption per capita of the household, $r(t)$ is the interest rate on assets, $w(t) L(t)$ is the flow of labor income earnings of the household and $r^{t}$ is supposed to be an effective tax rate on the rate of return from capital income. Put per capita assets as $a(t)=\mathbb{A}(t) / L(t)$, then it follows 
from (1), (2) and Itô formula that,

$$
d a(t)=\left[\left(1-r^{t}\right) r(t) a(t)+w(t)-c(t)-n a(t)+\sigma^{2} a(t)\right] d t=\sigma a(t) d B(t)
$$

On the other hand, we specifically adopt the following aggregate production function,

$$
Y(t)=A K(t)
$$

with $A>0$. Notice that this production function does not depend on labor, thus wage earnings, $w(t)$, in (3) will be equal to zero. Dividing both sides of this equation by $L(t)$, and as usual, define $k(t) \triangleq K(t) / L(t)$ as the capital-labor ratio, we obtain per capita output as

$$
f(k(t))=y(k(t)) \equiv y(t) \triangleq Y(t) / L(t)=A k(t)
$$

from which it is easily seen that output is only a function of capital, and there are no diminishing returns. What's more, the Inada conditions are no longer satisfied. In particular,

$$
\lim _{k(t) \rightarrow \infty} f^{\prime}(k(t))=A>0
$$

which is essential for sustained growth.

The conditions for profit-maximization require that the marginal product of capital be equal to the rental price of capital, $R(t)=r(t)+\delta$, in which $\delta$ is the depreciation rate. Since, as is obvious from equation (4), the marginal product of capital is constant and equal to $A$, thus $R(t)=A$ for all $t$, which implies that the net rate of return on the savings is constant and equal to

$$
r(t)=A-\delta, \quad \forall t \geq 0
$$

Next using the fact that $a(t)=k(t), w(t)=0, c(t)=\left(1-r^{s}\right) A k(t)$ and equation in (5), one can rewrite (3) as

$$
d k(t)=\left[r^{s} A-\delta-(A-\delta) r^{t}-n+\sigma^{2}\right] k(t) d t-\sigma k(t) d B(t)
$$

with $k(0) \triangleq k$ and $r^{s}$ denoting the saving rate. Then it follows that,

Lemma 1. There is some $e(p, T)<\infty$ such that

$$
\mathbb{E}\left[\sup _{0 \leq t \leq T}|k(t)|^{p}\right] \leq e(p, T)
$$

for $\forall 2 \leq p<\infty$ and $\forall 0<T<\infty$. 


\section{Proof. See Appendix A.}

Now, we consider the following special objective function,

$$
\int_{s}^{\tau^{*}} \exp (-\rho(t-s)) \ln [c(t)] d t+U^{\tau^{*}}
$$

where $0 \leq s<\tau^{*}$ and $\tau^{*}$ is an $\mathcal{F}_{t^{-}}$-stopping time, which with the term $U^{\tau^{*}}$ are determined by the following optimal stopping problem

$$
\begin{aligned}
g^{*}(\tau, k(\tau)) & \triangleq \sup _{\tau \in \mathcal{T}} \mathbb{E}^{(s, k)}\left[e^{-\rho \tau} \ln \left[\left(1-r^{s}\right) A k(\tau)\right] \mid \mathcal{F}_{\tau}\right] \\
& \left(=\sup _{\tau \in \mathcal{T}} \mathbb{E}^{(s, k)}\left[e^{-\rho \tau} \ln [c(\tau)] \mid \mathcal{F}_{\tau}\right)\right. \\
& =\mathbb{E}^{(s, k)}\left[e^{-\rho \tau^{*}} \ln \left[\left(1-r^{s}\right) A k\left(\tau^{*}\right)\right] \mid \mathcal{F}_{\tau^{*}}\right]
\end{aligned}
$$

subject to the stochastic differential equation in $(6)$, and $\mathcal{T} \triangleq\{\mathcal{F}$-stopping times $\}$. In what follows, we will calculate the optimal stopping time in a stochastic diffusion process.

Let $Y(t) \triangleq(s+t, k(t))^{T}$ and $Y(0)=(s, k(0))^{T} \triangleq(s, k)^{T}$, then the generator of $Y(t)$ is,

$$
\mathcal{A} \phi(s, k)=\frac{\partial \phi}{\partial s}+\left[r^{s} A-\delta-(A-\delta) r^{t}+\sigma^{2}-n\right] k \frac{\partial \phi}{\partial k}+\frac{1}{2} \sigma^{2} k^{2} \frac{\partial^{2} \phi}{\partial k^{2}}
$$

If we try a function $\phi$ of the form

$$
\phi(s, k)=e^{\rho s} k^{\lambda} \text { for some constant } \lambda \in \mathbb{R}
$$

we get

$$
\begin{aligned}
\mathcal{A} \phi(s, k) & =e^{-\rho s} k^{\lambda}\left\{-\rho+\left[r^{s} A-\delta-(A-\delta) r^{t}+\sigma^{2}-n\right] \lambda+\left[\sigma^{2} \lambda(\lambda-1) / 2\right]\right\} \\
& =e^{-\rho s} k^{\lambda} h(\lambda)
\end{aligned}
$$

in which

$$
h(\lambda) \triangleq\left(\sigma^{2} \lambda^{2} / 2\right)+\left[r^{s} A-\delta-(A-\delta) r^{t}+\left(\sigma^{2} / 2\right)-n\right] \lambda-\rho
$$

Solving equation $h(\lambda)=0$ gives the unique positive root,

$$
\lambda=\frac{\delta+(A-\delta) r^{t}+n-r^{s} A-\left(\sigma^{2} / 2\right)+\sqrt{\Delta}}{\sigma^{2}}
$$


where

$$
\Delta=\left[r^{s} A-\delta-(A-\delta) r^{t}+\left(\sigma^{2} / 2\right)-n\right]^{2}+2 \sigma^{2} \rho
$$

with this value of $\lambda$ we put

$$
\phi(s, k)= \begin{cases}e^{-\rho s} C k^{\lambda}, & (s, k) \in D \\ e^{-\rho s} \ln \left[\left(1-r^{s}\right) A k\right], & (s, k) \notin D\end{cases}
$$

for some constant $C$, to be determined. If we let $g(s, k) \triangle e^{-\rho s} \ln \left[\left(1-r^{s}\right) A k\right]$, we have

$$
\begin{aligned}
\mathcal{A} g(s, k) & =e^{-\rho s}\left\{-\rho \ln \left[\left(1-r^{s}\right) A k\right]+\left[r^{S} A-\delta-(A-\delta) r^{t}+\sigma^{2}-n\right]-\left(\sigma^{2} / 2\right)\right\} \\
& >0 \\
& \Longleftrightarrow k<\exp \left\{\left[r^{s} A-\delta-(A \delta) r^{t}-n+\left(\sigma^{2} / 2\right)\right] / \rho\right\} /\left[\left(1-r^{s}\right) A\right]
\end{aligned}
$$

Therefore,

$$
U=\left\{(s, k) ; k<\exp \left\{\left[r^{s} A-\delta-(A-\delta) r^{t}-n+\left(\sigma^{2} / 2\right)\right] / \rho\right\} /\left[\left(1-r^{s}\right) A\right]\right\}
$$

Thus, we guess that the continuation region $D$ has the form

$$
D=\left\{(s, k) ; 0<k<k^{*}\right\}
$$

for some $k^{*}$ such that $U \subseteq D$, i.e.,

$$
k^{*} \geq \exp \left\{\left[r^{s} A-\delta-(A-\delta) r^{t}-n+\left(\sigma^{2} / 2\right)\right] / \rho\right\} /\left[\left(1-r^{s}\right) A\right]
$$

Hence, by (13) we can rewrite (11) as

$$
\phi(s, k)= \begin{cases}e^{-\rho s} C k^{\lambda}, & 0<k<k^{*} \\ e^{-\rho s} \ln \left[\left(1-r^{s}\right) A k\right], & k \geq k^{*}\end{cases}
$$

for some constant $C>0$ (to be determined). We guess that the value function $\phi$ is $C^{1}$ at $k=k^{*}$ and this gives the following "high contact"conditions,

$$
C\left(k^{*}\right)^{\lambda}=\ln \left[\left(1-r^{s}\right) A k^{*}\right] \quad\left(\text { continuity at } k=k^{*}\right)
$$

and

$$
C \lambda\left(k^{*}\right)^{\lambda-1}=\left(k^{*}\right)^{-1} \quad\left(\text { differentiability at } k=k^{*}\right)
$$

Combining (16) with (17) one can get

$$
\begin{aligned}
\frac{C\left(k^{*}\right)^{\lambda}}{C \lambda\left(k^{*}\right)^{\lambda-1}} & =\frac{\ln \left[\left(1-r^{s}\right) A k^{*}\right]}{\left(k^{*}\right)^{-1}} \\
\Longleftrightarrow k^{*} & =[\exp (1 / \lambda)] /\left[\left(1-r^{s}\right) A\right]
\end{aligned}
$$


and

$$
C=\left(k^{*}\right)^{-\lambda} / \lambda=\left\{[\exp (1 / \lambda)] /\left[\left(1-r^{s}\right) A\right]\right\}^{-\lambda} / \lambda
$$

To summarize, then we get,

Theorem 1 (Endogenous Efficient Terminal Capital Stock). Under above assumptions and constructions, if $\sigma<0, \sigma^{2}<\rho$, and

$\delta+(A-\delta) r^{t}+n+\left(\sigma^{2} / 2\right)<\sigma^{2}+r^{s} A \leq \delta+n+(A-\delta) r^{t}+\rho-\left(\sigma^{2} / 2\right)$,

then we obtain the optimal $\mathcal{F}_{t}$-stopping time $\tau^{*}=\tau_{D} \triangleq \inf \{t \geq 0 ; k(t)=$ $\left.k^{*}\right\}$. In other words,

$$
g^{*}(s, k)=e^{-\rho s}\left(k^{*}\right)^{-\lambda} k^{\lambda} / \lambda=U^{\tau^{*}},
$$

which is a supermeanvalued majorant of $g(s, k)$ with $k^{*}$ and $\lambda$ are given by (18) and (10), respectively.

Proof. See Appendix B.

Remark 2.1. The theorem shows that the horizon of the economy and the terminal stock, which is also efficient capital accumulation in the sense of maximizing the discounted welfare function of the representative household referring to $\left(7^{\prime}\right)$, are endogenously determined. Next we will study the turnpike theorems in the stochastic growth model.

Theorem 2 (Local Uniform-Topology Turnpike Theorem). Given a complete filtered probability space $\left(\Omega, \mathcal{F},\left\{\mathcal{F}_{t}\right\}_{t \geq 0}, \mathbb{P}\right)$. If

$$
r^{s} A+\sigma^{2}=\delta+(A-\delta) r^{t}+n,
$$

then $k(t)$ is uniformly bounded for $t \in[0, T](\forall T>0)$ and for a.a.w, and furthermore $k(t)$ uniformly converges to $k^{*}$ for $t \in\left[0, \tau_{D}\right]$ and for a.a. $\omega$, where $\tau_{D}$ is the optimal stopping time defined in Theorem 1.

\section{Proof. See Appendix C.}

Now, we will provide some local characterizations of the efficient terminal capital stock by the following theorem.

Theorem 3 (Neighborhood Properties Of The Efficient Capital Stock). If $r^{S} A+\sigma^{2} \neq \delta+n+(A-\delta) r^{t}, k(t)$ will still be a local martingale on 
probability space $\left(\Omega, \mathcal{F}_{T}, \mathbb{Q}\right)(\forall T>0)$, where $\mathbb{Q}$ is equivalent to $\mathbb{P}$, and $k(t)$ is stochastically ultimately bounded. Moreover, there exists a constant $E>0$ and a Wiener measure $\nu$, defined on the canonical probability $s$ pace for Brownian motion, on Borel sigma algebra $\mathfrak{B}(C[0, \infty))$ generated by $(k(t) ; t \geq 0)$ such that

(i) $\mathbb{E}_{\mathbb{Q}}^{k}\left[\tau_{\bar{B}_{\alpha}\left(k^{*}\right)}\right] \leq \frac{\operatorname{dist}\left(k, k^{*}\right)}{\alpha^{2}-E}$,

(ii) $\lim \sup _{t \rightarrow \infty} \mathbb{E}_{\mathbb{Q}}^{k}\left\{\frac{1}{t} \int_{0}^{t}\left|k(s)-k^{*}\right|^{2} d s\right\} \leq E$,

(iii) $\nu\left\{B_{\alpha}\left(k^{*}\right)\right\} \geq 1-\frac{E}{\alpha^{2}}$, in which

$$
B_{\alpha}\left(k^{*}\right) \triangleq\left\{k(t) ;\left|k(t)-k^{*}\right|<\alpha, \forall t \geq 0\right\}, \tau_{\bar{B}_{\alpha}\left(k^{*}\right)} \triangleq \inf \left\{t ; k(t) \in \bar{B}_{\alpha}\left(k^{*}\right)\right\},
$$

And

$$
\operatorname{dist}\left(k, k^{*}\right) \triangleq k^{*} \log \left(k^{*} / k\right),
$$

which is the Kullback-Leibler distance between $k$ and $k^{*}$ with $E<\alpha^{2}, \forall \alpha>$ $0, \forall k(0)=k>0$ and $k^{*}$ is defined in (18).

\section{Proof. See Appendix D.}

Moreover, we can obtain the following turnpike theorem about capital accumulation, thereby extending the conclusion in Theorem 2 .

Theorem 4 (Uniform-Topology Turnpike Theorem 1). There exists some $C(p, T)>0$ such that

$$
\mathbb{E}\left[\sup _{0 \leq t \leq T}\left|k(t)-k^{*}\right|^{p}\right] \leq C(p, T) \text { for } \forall p>2 \text { and } \forall T>0
$$

Particularly, if $\sigma \rightarrow 0$, then we have

$$
\mathbb{E}\left[\lim _{T \rightarrow \infty} \sup _{0 \leq t \leq T}\left|k(t)-k^{*}\right|^{p}\right] \rightarrow 0
$$

Proof. See Appendix E.

Remark 2.2. This turnpike theorem implies that the path of capital accumulation will uniformly converge to the efficient capital stock, also the terminal capital stock, if the stochastic effect is sufficiently close to zero. And thus this theorem provides conditions under which the terminal capital 
stock is uniformly reachable, which is obviously much stronger than Joshi (1997)'s argument.

Now we consider the following stochastic optimal control problem facing the representative household,

$$
\max _{c(t)} \int_{s}^{\tau^{*}} \exp (-\rho(t-s)) \ln (c(t)) d t
$$

subject to

$$
d k(t)=\left\{\left[\left(1-r^{t}\right)(A-\delta)-n+\sigma^{2}\right] k(t)-c(t)\right\} d t-\sigma k(t) d B(t)
$$

We prove that there exists a continuously differential function $W(k(t))$, satisfying the following Bellman-Isaacs-Fleming differential equation,

$$
\begin{aligned}
& \rho W(k(t))-\frac{1}{2} \sigma^{2} k(t)^{2} W_{k k}(k(t)) \\
= & \max _{c(t)}\left(\ln (c(t))+W_{k}(k(t))\left\{\left[\left(1-r^{t}\right)(A-\delta)-n+\sigma^{2}\right] k(t)-c(t)\right\}\right)
\end{aligned}
$$

Applying the maximization operator, yields the following condition for a maximum as

$$
c^{*}(t)=1 / W_{k}(k(t))
$$

Substituting (21) into (20) produces

$$
\begin{aligned}
& \rho W(k(t))-\frac{1}{2} \sigma^{2} k(t)^{2} W_{k k}(k(t)) \\
= & -\ln \left[W_{k}(k(t))\right]+W_{k}(k(t))\left[\left(1-r^{t}\right)(A-\delta)-n+\sigma^{2}\right] k(t)-1
\end{aligned}
$$

Try

$$
W(k(t))=C_{1}+C_{2} \ln (k(t))
$$

for some constants $C_{1}, C_{2}$ to be determined. Then it is easy to get,

$$
C_{1}=\left\{\ln (\rho)-\left(\sigma^{2} \rho^{-1} / 2\right)+\rho^{-1}\left[\left(1-r^{t}\right)(A-\delta)-n+\sigma^{2}\right]-1\right\} / \rho
$$

And

$$
C_{2}=\rho^{-1}
$$

And hence by $(21)$

$$
\begin{aligned}
& c^{*}(t)=\rho k(t) \\
= & \rho k(0) \exp \left\{\left[r^{s} A-\delta-(A-\delta) r^{t}+\left(\sigma^{2} / 2\right)-n\right] t-\sigma B(t)\right\}
\end{aligned}
$$


Thus, in order to study the effect of temporary fiscal policy, i.e., capital income taxation, on equilibrium consumption path, we now define

$$
\begin{aligned}
\bar{c}^{*} & \triangleq c^{*}\left(t, \bar{r}_{0}^{t}\right)=\rho \bar{k}(t) \triangleq \rho k\left(t, \bar{r}_{0}^{t}\right), \\
\tilde{c}^{*} & \triangleq c^{*}\left(t, \tilde{r}_{0}^{t}\right)=\rho \tilde{k}(t) \triangleq \rho k\left(t, \tilde{r}_{0}^{t}\right), \\
\bar{k}(0) & \equiv \tilde{k}(0)
\end{aligned}
$$

where $\bar{r}_{0}^{t}$ and $\tilde{r}_{0}^{t}$ are two different temporary fiscal policies. Then we get the following theorem,

Theorem 5 (Inefficacy Of Temporary Fiscal Policy). If we choose $k(0)$ such that

$$
\begin{aligned}
& 2 T|\bar{\kappa}-\tilde{\kappa}|^{2} \frac{|k(0)|^{2}}{\tilde{\kappa}_{1}}\left(\exp \left(\tilde{\kappa}_{1} T\right)-1\right) e^{2\left(T|\bar{\kappa}|^{2}+\sigma^{2}\right) T} \leq \varepsilon / 3, \\
& \forall 0<T<\infty, \forall \varepsilon>0
\end{aligned}
$$

where

$$
\begin{aligned}
\bar{\kappa} & \triangleq r^{s} A-\delta-(A-\delta) \bar{r}_{0}^{t}+\sigma^{2}-n, \\
\tilde{\kappa} & \triangleq r^{s} A-\delta-(A-\delta) \tilde{r}_{0}^{t}+\sigma^{2}-n, \\
\tilde{\kappa}_{1} & \triangleq 2 r^{s} A-2 \delta-2(A-\delta) \tilde{r}_{0}^{t}+3 \sigma^{2}-2 n, \\
\sigma & \neq 0,
\end{aligned}
$$

Then we obtain,

$$
\mathbb{E}\left[\lim _{T \rightarrow \infty} \sup _{0 \leq t \leq T}\left|\bar{c}^{*}(t)-\tilde{c}^{*}(t)\right|^{2}\right] \rightarrow 0 \text { as } \varepsilon \rightarrow 0 .
$$

Proof. See Appendix F.

Remark 2.3. This theorem shows that, given two different temporary capital income taxation policies $\bar{r}_{0}^{t}$ and $\tilde{r}_{0}^{t}$, the distance between the corresponding equilibrium paths of consumption allocation, $\bar{c}^{*}(t)$ and $\tilde{c}^{*}(t)$, is arbitrarily small in the sense of mean-square uniform topology if the initial level of capital stock is sufficiently low, which differs from Yano (1998)'s requirement that the discount factor is sufficiently close to 1.

By Theorem 1, one can put,

$$
c^{*} \triangleq\left(1-r^{s}\right) A k^{*}
$$


And, by (22), Itô formula and (6), we get

$$
d c^{*}(t)=\rho\left[r^{s} A-\delta-(A-\delta) r^{t}-n+\sigma^{2}\right] k(t) d t-\rho \sigma k(t) d B(t)
$$

Then we get the following theorem,

Theorem 6 (Uniform-Topology Turnpike Theorem 2). There exists some $C(p, T)>0$ such that

$$
\mathbb{E}\left[\sup _{0 \leq t \leq T}\left|c^{*}(t)-c^{*}\right|^{p}\right] \leq C(p, T) \forall p \geq 2
$$

Moreover, if $\rho \rightarrow 0$ or $\sigma \rightarrow 0$, then we get

$$
\mathbb{E}\left[\lim _{T \rightarrow \infty} \sup _{0 \leq t \leq T}\left|c^{*}(t)-c^{*}\right|^{p}\right] \rightarrow 0 \quad \forall p \geq 2
$$

\section{Proof. See Appendix G.}

Remark 2.4. This turnpike theorem shows that the equilibrium consumption path will uniformly converge to the efficient consumption allocation of the dynamic equilibrium economy, conditioned on sufficiently small discount factor or stochastic effect. And it is easy to find out the difference between this turnpike theorem and those in Yano (1984a, 1984b, 1985), which, in stationary environments, require the discount factor sufficiently close to one.

Now we will prove the turnpike theorem for equilibrium allocation vector paths of the dynamic economy, and we define

$$
\begin{aligned}
& \Phi(t) \triangleq\left(-k(t) \quad y(t) \quad c^{*}(t)\right)^{T} \\
& \Phi^{*} \triangleq\left(-k^{*} y^{*} c^{*}\right)^{T}
\end{aligned}
$$


where $k^{*}$ is defined in (18), and $c^{*} \triangleq\left(1-r^{s}\right) A k^{*}=\left(1-r^{s}\right) y^{*}$. Then by (4), (6) and (23), we put

$$
\begin{aligned}
d \Phi(t) & =\left(\begin{array}{c}
-d k(t) \\
d y(t) \\
d c^{*}(t)
\end{array}\right)=\left(\begin{array}{c}
-\left[r^{s} A-\delta-(A-\delta) r^{t}-n+\sigma^{2}\right] \\
A\left[r^{s} A-\delta-(A-\delta) r^{t}-n+\sigma^{2}\right] \\
\rho\left[r^{s} A-\delta-(A-\delta) r^{t}-n+\sigma^{2}\right]
\end{array}\right) k(t) d t \\
& +\left(\begin{array}{c}
1 \\
-A \\
-\rho
\end{array}\right) \sigma k(t) d B(t) \\
& \triangleq\left(\begin{array}{c}
\bar{\zeta}_{1} \\
\bar{\zeta}_{2} \\
\bar{\zeta}_{3}
\end{array}\right) k(t) d t+\left(\begin{array}{c}
1 \\
-A \\
-\rho
\end{array}\right) \sigma k(t) d B(t)
\end{aligned}
$$

Then we obtain the following theorem,

Theorem 7 (Uniform-Topology Turnpike Theorem 3). There exists some $C(p, T)>0$ such that

$$
\mathbb{E}\left[\sup _{0 \leq t \leq T}\left\|\phi(t)-\Phi^{*}\right\|_{p}^{p}\right] \leq C(p, T) \forall p \geq 2,
$$

Moreover, if $\sigma \rightarrow 0$, then we have

$$
\mathbb{E}\left[\lim _{T \rightarrow \infty} \sup _{0 \leq t \leq T}\left\|\phi(t)-\Phi^{*}\right\|_{p}^{p}\right] \rightarrow 0 \forall p \geq 0
$$

where \|\|$_{p}$ denotes $L^{p}$-norm.

\section{Proof. See Appendix H.}

Remark 2.5. The economic intuition of this turnpike theorem is that the equilibrium allocation vector path of the dynamic economy will uniformly converge to the efficient allocation vector including capital, output and consumption, when the stochastic effect is sufficiently small. And, what's more, we can easily see that this turnpike theorem does not depend on the constraint of discount factor like those turnpike theorems proved in Yano (1984a, 1984b, 1985).

\section{CONCLUDING REMARKS}

In the paper, stochastic versions of turnpike theorems have been established in a stochastic endogenous growth model and the inefficacy of 
temporary fiscal policy which is specifically chosen to be capital income taxation has also been demonstrated under relatively weak conditions. To summarize, there are three novelties in the paper: first, we provide a possible way making the horizon of the economy and the terminal capital stock, also efficient capital accumulation in some sense, all endogenously determined; second, we prove that a single welfare function in an aggregated model of optimal growth can also be endogenously defined as is shown in decentralized models; third, we prove much stronger turnpike theorems under uncertainty and in the sense of uniform topology, which we call uniform-topology turnpike theorems.

Obviously, the present study can be easily extended at least from the following three directions: first, jump diffusion process like Itô-Lévy process can be introduced into stochastic optimal growth models; second, more complicated and more comprehensive method, say, integro-variational inequalities for optimal stopping problems (see, Øksendal and Sulem, 2007) in stochastic analysis, making the horizon, the terminal stock and further the welfare function of the abstract economy endogenously determined, can be reasonably employed; third, the methodology of studies on turnpike theorems can be naturally extended to investigate the distance and the convergence between different economical systems, when their evolutionary or development paths are abstractly determined by different differential equations, ordinary or stochastic, of capitals, including physical capital, environmental capital and also human capital.

\section{Proof of Lemma 1}

\section{APPENDIX A}

Since by (6)

$$
d k(t)=f(k(t)) d t+g(k(t)) d B(t)
$$

where

$$
\begin{gathered}
f(k(t)) \triangleq\left[r^{s} A-\delta-(A-\delta) r^{t}+\sigma^{2}-n\right] k(t) \triangleq \varpi k(t) \\
g(k(t)) \triangleq-\sigma k(t)
\end{gathered}
$$

Then by the Itô formula,

$$
\begin{aligned}
|k(t)|^{2} & =|k(0)|^{2}+2 \int_{0}^{t}\langle f(k(s)), k(s)\rangle d s \\
& +\int_{0}^{t}|g(k(s))|^{2} d s+2 \int_{0}^{t}\langle k(s), g(k(s)) d B(s)\rangle
\end{aligned}
$$


where $\langle\cdot, \cdot\rangle$ denotes standard inner product. Choose some $\gamma$ such that,

$$
|\langle f(k(t)), k(t)\rangle| \bigvee|g(k(t))|^{2} \leq(\gamma+\sigma)^{2}|k(t)|^{2}
$$

Thus for some $e=e(p)$ and $t_{1} \in[0, T]$,

$$
\begin{aligned}
\sup _{0 \leq t \leq t_{1}}|k(t)|^{p} & \leq e\left\{|k(0)|^{p}+\left(\int_{0}^{t_{1}}(\sigma+\gamma)^{2}|k(s)|^{2} d s\right)^{p / 2}\right. \\
& \left.+\sup _{0 \leq t \leq t_{1}}\left|\int_{0}^{t}\langle k(s), g(k(s)) d B(s)\rangle\right|^{p / 2}\right\}
\end{aligned}
$$

It follows from Cauchy-Schwarz inequality that

$$
\begin{aligned}
\sup _{0 \leq t \leq t_{1}}|k(t)|^{p} & \leq e\left(|k(0)|^{p}+|\sigma+\gamma|^{p} T^{(p-2) / 2} \int_{0}^{t_{1}}|k(s)|^{p} d s\right. \\
& \left.+\sup _{0 \leq t \leq t_{1}}\left|\int_{0}^{t}\langle k(s), g(k(s)) d B(s)\rangle\right|^{p / 2}\right)
\end{aligned}
$$

Taking expectations and for $|\sigma+\gamma|>T^{(2-p) / 2 p}$, we have

$$
\begin{aligned}
\mathbb{E}\left[\sup _{0 \leq t \leq t_{1}}|k(t)|^{p}\right] \leq & e|\sigma+\gamma|^{p} T^{(p-2) / 2}\left\{\mathbb{E}|k(0)|^{p}+\mathbb{E} \int_{0}^{t_{1}}|k(s)|^{p} d s\right. \\
& \left.+\mathbb{E}\left[\sup _{0 \leq t \leq t_{1}}\left|\int_{0}^{t}\langle k(s), g(k(s)) d B(s)\rangle\right|^{p / 2}\right]\right\}
\end{aligned}
$$

Applying the Burkholder-Davis-Gundy inequality (see, Karatzas and Shreve, 1991, pp.166), and for some $\tilde{e}=\tilde{e}(p)$,

$$
\begin{aligned}
& \mathbb{E}\left[\sup _{0 \leq t \leq t_{1}}|k(t)|^{p}\right] \leq \tilde{e}|\sigma+\gamma|^{p} T^{(p-2) / 2}\left\{\mathbb{E}|k(0)|^{p}+\int_{0}^{t_{1}} \mathbb{E}|k(s)|^{p} d s\right. \\
& \left.+\mathbb{E}\left[\int_{0}^{t_{1}}|k(s)|^{2}|g(k(s))|^{2} d s\right]^{p / 4}\right\}
\end{aligned}
$$


Next, by the Young inequality (see, Higham et al, 2003) and Hölder inequality,

$$
\begin{aligned}
& \mathbb{E}\left[\int_{0}^{t}|k(s)|^{2}|g(k(s))|^{2} d s\right]^{p / 4} \leq \mathbb{E}\left[\sup _{0 \leq s \leq t_{1}}|k(s)|^{p / 2}\left(\int_{0}^{t_{1}}|g(k(s))|^{2} d s\right)^{p / 4}\right] \\
\leq & \frac{1}{2 \tilde{e}|\sigma+\gamma|^{p} T^{(p-2) / 2}} \mathbb{E}\left[\sup _{0 \leq s \leq t_{1}}|k(s)|^{p}\right]+\frac{\tilde{e}|\sigma+\gamma|^{p} T^{(p-2) / 2}}{2} \mathbb{E}\left[\int_{0}^{t_{1}}|g(k(s))|^{2} d s\right]^{p / 2} \\
\leq & \frac{1}{2 \tilde{e}|\sigma+\gamma|^{p} T^{(p-2) / 2}} \mathbb{E}\left[\sup _{0 \leq s \leq t_{1}}|k(s)|^{p}\right]+\frac{\tilde{e}}{2}|\sigma+\gamma|^{p}|\sigma|^{p} T^{p-2} \mathbb{E}\left[\int_{0}^{t_{1}}|k(s)|^{p} d s\right]
\end{aligned}
$$

Substituting this into (A.1) yields,

$$
\begin{aligned}
\mathbb{E}\left[\sup _{0 \leq t \leq t_{1}}|k(t)|^{p}\right] \leq & 2 \tilde{e}|\sigma+\gamma|^{p} T^{(p-2) / 2}\left\{\mathbb{E}|k(0)|^{p}+\int_{0}^{t_{1}} \mathbb{E}|k(s)|^{p} d s\right. \\
& \left.+\frac{\left.\tilde{e}|\sigma+\gamma|\right|^{p}|\sigma|^{p} T^{p-2}}{2} \mathbb{E}\left[\int_{0}^{t_{1}}|k(s)|^{p} d s\right]\right\}
\end{aligned}
$$

If $\left(\tilde{e}|\sigma+\gamma|^{p}|\sigma|^{p} T^{p-2}\right) / 2 \geq 1$, then for some $\bar{e}=\bar{e}(p)$,

$$
\begin{aligned}
\mathbb{E}\left[\sup _{0 \leq t \leq T}|k(t)|^{p}\right] & \leq|\sigma+\gamma|^{2 p}|\sigma|^{p} T^{3(p-2) / 2} \bar{e}\left(\mathbb{E}|k(0)|^{p}+\int_{0}^{t_{1}} \mathbb{E}|k(s)|^{p} d s\right) \\
& \leq|\sigma+\gamma|^{2 p}|\sigma|^{p} T^{3(p-2) / 2} \bar{e}(p)\left(|k(0)|^{p}+\frac{|k(0)|^{p}}{\hat{e}}[\exp (\hat{e} T)-1]\right)
\end{aligned}
$$

in which

$$
\hat{e}=\hat{e}(p) \triangleq p\left[r^{s} A-\delta-(A-\delta) r^{t}+\left(\sigma^{2} / 2\right)-n\right]+\left(p^{2} \sigma^{2} / 2\right)
$$

Given $k(0)$, there is some $e(p, T)<\infty$ such that

$$
\mathbb{E}\left[\sup _{0 \leq t \leq T}|k(t)|^{p}\right] \leq e(p, T)
$$

\section{Proof of Theorem 1}

\section{APPENDIX B}

By the Theorem in Øksendal (2003), pp.224-226, it is easy to see that we just need to prove the following cases,

(i) We need to prove that $\phi \geq g$ on $D$, i.e., that

$$
C k^{\lambda} \geq \ln \left[\left(1-r^{s}\right) A k\right] \text { for } 0<k<k^{*}
$$


Define $l(k) \triangleq C k^{\lambda}-\ln \left[\left(1-r^{s}\right) A k\right]$. By our chosen values of $C$ and $k^{*}$ we have $l\left(k^{*}\right)=l^{\prime}\left(k^{*}\right)=0$. Moreover, since $l^{\prime \prime}(k)=C \lambda(\lambda-1) k^{\lambda-2}+k^{-2}$, if we put $\lambda>1$, then $l^{\prime \prime}(k)>0$ for $0<k<k^{*}$ and thus we have $l(k)>0$ for all $0<k<k^{*}$. By $(10)$,

$$
\begin{aligned}
& \lambda>1 \\
\Leftrightarrow & \sqrt{\left[r^{s} A-\delta-(A-\delta) r^{t}+\left(\sigma^{2} / 2\right)-n\right]^{2}+2 \sigma^{2} \rho} \\
& >\sigma^{2}+\left[r^{s} A-\delta-(A-\delta) r^{t}+\left(\sigma^{2} / 2\right)-n\right]
\end{aligned}
$$

If

$$
r^{s} A+\left(3 \sigma^{2} / 2\right) \leq \delta+(A-\delta) r^{t}+n,
$$

Then $\lambda>1$ always holds. Otherwise, put

$$
r^{s} A+\sigma^{2}>\delta+n+(A-\delta) r^{t}-\left(\sigma^{2} / 2\right)
$$

Then,

$$
\begin{aligned}
& \lambda>1 \\
\Leftrightarrow & {\left[r^{s} A-\delta+\left(\sigma^{2} / 2\right)-(A-\delta) r^{t}-n\right]^{2}+2 \sigma^{2} \rho } \\
> & \left\{\sigma^{2}+\left[r^{s} A-\delta-(A-\delta) r^{t}+\left(\sigma^{2} / 2\right)-n\right]\right\}^{2} \\
\Leftrightarrow & \rho+\delta+(A-\delta) r^{t}+n>\sigma^{2}+r^{s} A
\end{aligned}
$$

Thus,

$$
\lambda>1 \text { when } r^{s} A+\left(3 \sigma^{2} / 2\right) \leq \delta+n+(A-\delta) r^{t}
$$

or

$\lambda>1$ when $\rho+\delta+(A-\delta) r^{t}+n>\sigma^{2}+r^{s} A>\delta+n+(A-\delta) r^{t}-\left(\sigma^{2} / 2\right) \quad($ B.2)

To sum up, either (B.1) or (B.2) can make (i) hold true.

(ii) Outside $D$ we have $\phi(s, k)=e^{-\rho s} \ln \left[\left(1-r^{s}\right) A k\right]$ and therefore

$$
\begin{aligned}
\mathcal{A} \phi(s, k) & =e^{-\rho s}\left\{-\rho \ln \left[\left(1-r^{s}\right) A k\right]+\left[r^{s} A-\delta-(A-\delta) r^{t}+\left(\sigma^{2} / 2\right)-n\right]\right\} \\
& \leq 0 \text { for } \forall k \geq k^{*} \\
& \Leftrightarrow k \geq \exp \left\{\left[r^{s} A-\delta-(A-\delta) r^{t}-n+\left(\sigma^{2} / 2\right)\right] / \rho\right\} /\left[\left(1-r^{s}\right) A\right], \forall k \geq k^{*} \\
& \Leftrightarrow k^{*} \geq \exp \left\{\left[r^{s} A-\delta-(A-\delta) r^{t}-n+\left(\sigma^{2} / 2\right)\right] / \rho\right\} /\left[\left(1-r^{s}\right) A\right]
\end{aligned}
$$

which holds by (14).

(iii) To check if $\tau_{D}<\infty$ a.s, we consider the solution $k(t)$ of (6). First, we define

$$
G(t) \triangleq \ln [k(t)]
$$


Then by Itô formula,

$$
d G(t)=\left[r^{s} A-\delta-(A-\delta) r^{t}+\left(\sigma^{2} / 2\right)-n\right] d t-\sigma d B(t)
$$

Hence,

$$
G(t)=G(0)+\left[r^{s} A-\delta-(A-\delta) r^{t}+\left(\sigma^{2} / 2\right)-n\right] t-\sigma B(t)
$$

And this gives the solution

$$
k(t)=k \exp \left\{\left[r^{s} A-\delta-(A-\delta) r^{t}+\left(\sigma^{2} / 2\right)-n\right] t-\sigma B(t)\right\}
$$

We see that if

$$
r^{s} A+\left(\sigma^{2} / 2\right)>\delta+(A-\delta) r^{t}+n
$$

And

$$
\sigma<0
$$

Then,

$$
\lim _{t \rightarrow \infty} k(t)=\infty \quad \text { a.s. }
$$

by the law of the iterated logarithm of Brownian motion. And in particular $\tau_{D}<\infty$ a.s.,as required.

Remark B.1. A comparison of (B.2) and (B.4) shows that we must put

$$
\rho>\left(\sigma^{2} / 2\right)
$$

(iv) Since $\phi$ is bounded on $\left[0, k^{*}\right]$, it suffices to check that

$$
\left\{e^{-\rho \tau} \ln \left[\left(1-r^{s}\right) A k(\tau)\right]\right\}_{\tau \in \mathcal{T}} \text { is uniformly integrable on }\left[k^{*}, \infty\right)
$$

For this to hold it suffices that there exists a constant $M$ such that

$$
\mathbb{E}\left\{e^{-2 \rho \tau}\left[\ln \left(\left(1-r^{s}\right) A k(\tau)\right)\right]^{2}\right\} \leq M \text { for all } \tau \in \mathcal{T} \text { and } k(\tau) \geq k^{*}
$$

Since

$$
0<\ln \left[\left(1-r^{s}\right) A k(t)\right]<\left(1-r^{s}\right) A k(t) \text { on }\left[k^{*}, \infty\right)
$$

Hence by (B.3) we have

$$
\begin{aligned}
& \mathbb{E}\left\{e^{-2 \rho \tau}\left[\ln \left(\left(1-r^{s}\right) A k(\tau)\right)\right]^{2}\right\} \leq \mathbb{E}\left\{e^{-2 \rho \tau}\left[\left(1-r^{s}\right) A k(\tau)\right]^{2}\right\} \\
= & \left(1-r^{s}\right)^{2} A^{2} k^{2} \mathbb{E}\left[\exp \left\{\left[2 r^{s} A-2 \delta-2(A-\delta) r^{t}+\sigma^{2}-2 n-2 \rho\right] \tau-2 \sigma B(\tau)\right\}\right] \\
= & \left(1-r^{s}\right)^{2} A^{2} k^{2} \mathbb{E}\left[\exp \left\{\left[2 r^{s} A-2 \delta-2(A-\delta) r^{t}+3 \sigma^{2}-2 n-2 \rho\right] \tau\right\}\right]
\end{aligned}
$$


We conclude that if

$$
r^{s} A+\left(3 \sigma^{2} / 2\right) \leq \delta+n+\rho+(A-\delta) r^{t}
$$

the desired result is then immediate.

Remark B.2. A comparison of (B.4) and (B.7) shows that we must put

$$
\rho>\sigma^{2}
$$

\section{APPENDIX C}

\section{Proof of Theorem 2}

By (6), we have

$$
k(t)=k \exp \left\{\left[r^{s} A-\delta-(A-\delta) r^{t}+\left(\sigma^{2} / 2\right)-n\right] t-\sigma B(t)\right\}
$$

Let $\bar{\sigma} \triangleq-\sigma$, we have

$$
k(t)=k \exp \left\{\left[r^{s} A-\delta-(A-\delta) r^{t}+\left(\bar{\sigma}^{2} / 2-n\right] t+\bar{\sigma} B(t)\right\}\right.
$$

Put

$$
r^{s} A-\delta-(A-\delta) r^{t}+\left(\bar{\sigma}^{2} / 2\right)-n=-\bar{\sigma}^{2} / 2
$$

Then

$$
r^{s} A+\sigma^{2}=\delta+(A-\delta) r^{t}+n
$$

Hence, with $B_{t} \triangleq B(t)$, we have

$$
k(t)=k \exp \left\{\bar{\sigma} B_{t}-\left(\bar{\sigma}^{2} t / 2\right)\right\}
$$

Let $t \geq s \geq 0$, one can find

$$
\begin{aligned}
\mathbb{E}\left[k(t) \mid \mathcal{F}_{s}\right] & =k \mathbb{E}\left[\exp \left\{\bar{\sigma} B_{t}-\left(\bar{\sigma}^{2} t / 2\right)\right\} \mid \mathcal{F}_{s}\right] \\
& =k \exp \left\{\bar{\sigma} B_{s}-\left(\bar{\sigma}^{2} t / 2\right)\right\} \mathbb{E}\left[\exp \left\{\bar{\sigma}\left(B_{t}-B_{s}\right)\right\} \mid \mathcal{F}_{s}\right] \\
& =k \exp \left\{\bar{\sigma} B_{s}-\left(\bar{\sigma}^{2} t / 2\right)\right\} \mathbb{E}\left[\exp \left\{\bar{\sigma}\left(B_{t}-B_{s}\right)\right\}\right] \\
& =k \exp \left\{\bar{\sigma} B_{s}-\left(\bar{\sigma}^{2} t / 2\right)\right\} \int_{\mathbb{R}} \frac{\exp \left\{\bar{\sigma} x-\left[x^{2} / 2(t-s)\right]\right\}}{\sqrt{2 \pi(t-s)}} d \mu(x) \\
& =k \exp \left\{\bar{\sigma} B_{s}-\left(\bar{\sigma}^{2} t / 2\right)\right\} \exp \left\{\left(\bar{\sigma}^{2} / 2\right)(t-s)\right\} \\
& \times \int_{\mathbb{R}} \frac{\exp \left\{-[x-\bar{\sigma}(t-s)]^{2} / 2(t-s)\right\}}{\sqrt{2 \pi(t-s)}} d \mu(x) \\
& =k \exp \left\{\bar{\sigma} B_{s}-\left(\bar{\sigma}^{2} s / 2\right)\right\} \\
& =k(s)
\end{aligned}
$$


with $\mu$ the canonical Lebesgue-Stieltjes measure. Hence, $k(t)$ is an $\mathcal{F}_{t^{-}}$ martingale w.r.t.P. On the other hand, noting that by (C.1)

$$
\begin{aligned}
\mathbb{E}[|k(t)|] & =k \mathbb{E}\left[\exp \left\{\left[r^{s} A-\delta-(A-\delta) r^{t}+\left(\sigma^{2} / 2\right)-n\right] t-\sigma B(t)\right\}\right] \\
& =k \exp \left\{\left[r^{s} A-\delta-(A-\delta) r^{t}+\sigma^{2}-n\right] t\right\} \\
& =k \triangleq k(0)<\infty
\end{aligned}
$$

Thus, by the Doob's martingale inequality,

$$
\mathbb{P}\left\{\sup _{0 \leq t \leq T}|k(t)| \geq \lambda\right\} \leq \frac{1}{\lambda} \mathbb{E}[|k(T)|]=\frac{k}{\lambda}, \forall \lambda>0, \forall T>0
$$

Without loss of generality, we put $\lambda=2^{m}$ for $m \in \mathbb{N}$, then,

$$
\mathbb{P}\left\{\sup _{0 \leq t \leq T}|k(t)| \geq 2^{m}\right\} \leq \frac{1}{2^{m}} k, \forall m \in \mathbb{N}
$$

By the Borel-Cantelli lemma,

$$
\mathbb{P}\left\{\sup _{0 \leq t \leq T}|k(t)| \geq 2^{m} \text { for infinitely many } m\right\}=0
$$

So for a.a. $\omega$ there exists $\bar{m}(\omega)$ such that

$$
\sup _{0 \leq t \leq T}|k(t)|<2^{m} \text { for } m \geq \bar{m}(\omega)
$$

Thus,

$$
\limsup _{T \rightarrow \infty} \sup _{0 \leq t \leq T}|k(t)|<2^{m} \text { for } m \geq \bar{m}(\omega)
$$

Consequently, $k(t)=k(t, \omega)$ is uniformly bounded for $t \in[0, T](\forall T>0)$ and for a.a.w. Moreover, it is easily seen that $k(t)-k^{*}$ is also an $\mathcal{F}_{t^{-}}$ martingale. So, applying Doob's martingale inequality again, we obtain,

$$
\mathbb{P}\left\{\sup _{0 \leq t \leq T}\left|k(t)-k^{*}\right| \geq \varepsilon\right\} \leq \frac{\mathbb{E}\left[\left|k(T)-k^{*}\right|\right]}{\varepsilon}, \forall \varepsilon>0, \forall T>0
$$

Using the definition of $\tau_{D}$ in Theorem 1, we see that there exists $\alpha>0$ such that the above martingale inequality still holds for $\forall t \in B_{\alpha}\left(\tau_{D}\right) \triangleq$ $\left\{t ;\left|t-\tau_{D}\right|<\alpha\right\}$. Without loss of generality, we set $\alpha=2^{-m}, \forall m \in \mathbb{N}$. Hence, $\forall T_{m} \in B_{\alpha}\left(\tau_{D}\right)$ and according to the continuity of martingale w.r.t. $t$ (given $\omega$ ), condition (C.2) and Lebesgue bounded convergence theorem, we have

$$
\limsup _{m \rightarrow \infty} \mathbb{P}\left\{\sup _{0 \leq t \leq T_{m}}\left|k(t)-k^{*}\right| \geq \varepsilon\right\} \leq \frac{\lim \sup _{m \rightarrow \infty} \mathbb{E}\left[\left|k\left(T_{m}\right)-k^{*}\right|\right]}{\varepsilon}=0 . \text { a.s. }
$$


which yields

$$
\limsup _{m \rightarrow \infty} \mathbb{P}\left\{\sup _{0 \leq t \leq T_{m}}\left|k(t)-k^{*}\right|<\varepsilon\right\} \geq 1 \text { a.s. }
$$

Letting $\varepsilon=2^{-i}, \forall i \in \mathbb{N}$, we get

$$
\limsup _{m \rightarrow \infty} \mathbb{P}\left\{\sup _{0 \leq t \leq T_{m}}\left|k(t)-k^{*}\right|<2^{-i}\right\}=1 \forall i \in \mathbb{N} \text { a.s. }
$$

It follows from Fatou lemma that,

$$
\mathbb{P}\left\{\sup _{0 \leq t \leq \tau_{D}}\left|k(t)-k^{*}\right|<2^{-i}\right\}=1 \forall i \in \mathbb{N} \text { a.s. }
$$

Thus, by the Borel-Cantelli lemma,

$$
\mathbb{P}\left\{\sup _{0 \leq t \leq \tau_{D}}\left|k(t)-k^{*}\right|<2^{-i} \text { for infinitely many } i\right\}=1
$$

So for a.a. $\omega$ there exists $\underline{i}(\omega)$ such that

$$
\sup _{0 \leq t \leq \tau_{D}}\left|k(t)-k^{*}\right|<2^{-i} \text { for } i \geq \underline{i}(\omega)
$$

Therefore, $k(t)$ uniformly converges to $k^{*}$ for $t \in\left[0, \tau_{D}\right]$ and for a.a.w.

\section{Proof of Theorem 3}

\section{APPENDIX D}

Note from Theorem 2 that $k(t)$ will not be a martingale on probability space $\left(\Omega, \mathcal{F}_{T}, \mathbb{P}\right)$ for $\forall T>0$ when $r^{s} A+\sigma^{2} \neq \delta+n+(A-\delta) r^{t}$. Since,

$$
d k(t)=b(t, \omega) d t+\sigma(t, \omega) d B(t)
$$

where

$$
\begin{aligned}
b(t, \omega) & \triangleq\left[r^{s} A-\delta-(A-\delta) r^{t}+\sigma^{2}-n\right] k(t) \\
\sigma(t, \omega) & \triangleq-\sigma k(t) \\
B(0) & \triangleq 0 \mathbb{P} \text { a.s. }
\end{aligned}
$$

We now put

$\theta(t) \triangle \frac{b(t, \omega)}{\sigma(t, \omega)}=\frac{\delta+n+(A-\delta) r^{t}-r^{s} A-\sigma^{2}}{\sigma} \triangle \theta$, for a.a. $(t, \omega) \in[0, T] \times \Omega$ 
Then,

$$
\begin{aligned}
Z(t) & \triangleq \exp \left\{-\int_{0}^{t} \theta(s) d B(s)-\frac{1}{2} \int_{0}^{t} \theta^{2}(s) d s\right\} \\
& =\exp \left(-\theta B(t)-\left(\theta^{2} t / 2\right)\right)
\end{aligned}
$$

Define a measure $\mathbb{Q}$ on $\mathcal{F}_{T}$ by,

$$
d \mathbb{Q}(\omega)=Z(T) d \mathbb{P}(\omega)
$$

i.e., $Z(T)$ is the so-called Radon-Nikodym derivative. Since,

$$
\begin{aligned}
\mathbb{E}_{\mathbb{P}}[Z(T)] & =\mathbb{E}_{\mathbb{P}}\left[\exp \left\{-\theta B(T)-\left(\theta^{2} T / 2\right)\right\}\right] \\
& =\exp \left\{\theta^{2} T / 2-\left(\theta^{2} T / 2\right)\right\} \\
& =1
\end{aligned}
$$

which shows, according to Girsanov theorem, that $\mathbb{Q}$ is a probability measure on $\mathcal{F}_{T}, \mathbb{Q}$ is equivalent to $\mathbb{P}$ and $k(t)$ is a local martingale w.r.t. $\mathbb{Q}$. Moreover,

$$
\mathbb{E}_{\mathbb{P}}\left[\exp \left((1 / 2) \int_{0}^{T} \theta^{2}(s) d s\right)\right]=\exp \left(\theta^{2} T / 2\right)<\infty \text { for } 0 \leq T<\infty
$$

which satisfies the Novikov condition. Using Girsanov theorem again, we conclude that the following process

$$
\hat{B}(t) \triangleq \int_{0}^{t} \theta(s) d s+B(t)=\theta t+B(t), \quad 0 \leq t \leq T
$$

is a Brownian motion w.r.t. $\mathbb{Q}$ with $\hat{B}(0)=B(0)=0$ a.s. and expressed in terms of $\hat{B}(t)$ we can get

$$
d k(t)=-\sigma k(t) d \hat{B}(t), \quad 0 \leq t \leq T
$$

Thus, it is easily seen that

$$
k(t)=k(0) \exp \left\{-\sigma \hat{B}(t)-\left(\sigma^{2} t / 2\right)\right\}
$$

which is defined on the measure space $\left(\Omega, \mathcal{F}_{T}, \mathbb{Q}\right)$. Then,

$$
\mathbb{E}_{\mathbb{Q}}[|k(t)|]=k \mathbb{E}_{\mathbb{Q}}\left[\exp \left\{-\sigma \hat{B}(t)-\left(\sigma^{2} t / 2\right)\right\}\right]=k
$$


and

$$
\begin{aligned}
\mathbb{E}_{\mathbb{Q}}[\sqrt{|k(t)|}] & =\sqrt{k} \mathbb{E}_{\mathbb{Q}}\left[\exp \left\{-(\sigma \hat{B}(t) / 2)-\left(\sigma^{2} t / 4\right)\right\}\right] \\
& =\sqrt{k} \exp \left(-\sigma^{2} t / 8\right)
\end{aligned}
$$

Thus,

$$
\lim _{t \rightarrow \infty} \mathbb{E}_{\mathbb{Q}}[\sqrt{|k(t)|}]=0
$$

Now for any $\varepsilon>0$ and any constant $H>0$, by the Chebyshev's inequality,

$$
\mathbb{Q}\{|k(t)|>H\} \leq \frac{\mathbb{E}_{\mathbb{Q}}[\sqrt{|k(t)|}]}{\sqrt{H}}
$$

Hence,

$$
\limsup _{t \rightarrow \infty} \mathbb{Q}\{|k(t)|>H\} \leq 0
$$

which implies

$$
\limsup _{t \rightarrow \infty} \mathbb{Q}\{|k(t)| \leq H\}=1
$$

Therefore, $k(t)$ is stochastically ultimately bounded. Now we define $M(t) \triangleq$ $k(t)-k^{*}$, also a $\mathbb{Q}$-local martingale, satisfying

$$
|M(t)|=\left|k(t)-k^{*}\right| \leq k(t)+k^{*}
$$

Hence,

$$
\begin{aligned}
\lim _{t \rightarrow \infty}|M(t)| & \leq \lim _{t \rightarrow \infty}\left[k \exp \left\{-\sigma \hat{B}(t)-\left(\sigma^{2} t / 2\right)\right\}\right]+k^{*} \\
& =\lim _{t \rightarrow \infty}\left\{k \exp \left[(\hat{B}(t) / t)(-\sigma t)-\left(\sigma^{2} t / 2\right)\right]\right\}+k^{*} \\
& =0+k^{*}=k^{*} \quad \text { a.s. }
\end{aligned}
$$

by the strong law of large numbers for martingale and the fact $0 \times \infty=0$. Hence,

$$
\lim _{t \rightarrow \infty}|M(t)|<+\infty \text { a.s. }
$$

For any integer $i \geq 1$, define the stopping time (or Markov time),

$$
\tau_{i} \triangleq \inf \{t \geq 0 ;|M(t)| \geq i\}
$$

Clearly, $\tau_{i} \uparrow \infty$ a.s., and $\mathbb{Q}(\bar{\Omega})=1$, where

$$
\bar{\Omega} \triangleq \bigcup_{i=1}^{\infty}\left\{\omega ; \tau_{i}(\omega)=\infty\right\}
$$


Note that for any $t \geq 0$,

$$
\mathbb{E}_{\mathbb{Q}}\left[\left|M\left(t \wedge \tau_{i}\right)\right|\right] \leq i
$$

Letting $t \rightarrow \infty$ and using Fatou lemma, we obtain

$$
\limsup _{t \rightarrow \infty} \mathbb{E}_{\mathbb{Q}}\left[\left|M\left(t \wedge \tau_{i}\right)\right|\right] \leq \mathbb{E}_{\mathbb{Q}}\left[\limsup _{t \rightarrow \infty}\left[\left|M\left(t \wedge \tau_{i}\right)\right|\right]\right]=\mathbb{E}_{\mathbb{Q}}\left[\left|M\left(\tau_{i}\right)\right|\right] \leq i
$$

Thus,

$$
\mathbb{E}_{\mathbb{Q}}\left[\left|k\left(\tau_{i}\right)-k^{*}\right|\right] \leq i<\infty
$$

Since $k\left(t \wedge \tau_{i}\right)-k^{*}$ is a $\mathbb{Q}$-martingale, thus by (D.1) and the Doob's martingale inequality,

$$
\begin{aligned}
\mathbb{Q}\left\{\omega ; \sup _{0 \leq t \leq T}\left|k\left(t \wedge \tau_{i}\right)-k^{*}\right| \geq \lambda\right\} & \leq \frac{\mathbb{E}_{\mathbb{Q}}\left[\left|k\left(T \wedge \tau_{i}\right)-k^{*}\right|\right]}{\lambda} \\
& \leq \frac{\mathbb{E}_{\mathbb{Q}}\left[\left|k\left(T \wedge \tau_{i}\right)\right|\right]+k^{*}}{\lambda} \\
& =\frac{k+k^{*}}{\lambda} \forall \lambda, T>0
\end{aligned}
$$

On the other hand, by Kolmogorov's inequality, we have

$$
\mathbb{Q}\left\{\omega ; \sup _{0 \leq t \leq T}\left|k\left(t \wedge \tau_{i}\right)-k^{*}\right| \geq \lambda\right\} \leq \frac{\operatorname{var}_{\mathbb{Q}}\left[\left|k\left(T \wedge \tau_{i}\right)-k^{*}\right|\right]}{\lambda^{2}}, \forall \lambda, T>0
$$

Hence, we have

$$
\begin{aligned}
\frac{\operatorname{var}_{\mathbb{Q}}\left[\left|k\left(T \wedge \tau_{i}\right)-k^{*}\right|\right]}{\lambda^{2}} & \leq \frac{k+k^{*}}{\lambda} \forall \lambda, T>0 \\
\Leftrightarrow \operatorname{var}_{\mathbb{Q}}\left[\left|k\left(T \wedge \tau_{i}\right)-k^{*}\right|\right] & \leq\left(k+k^{*}\right) \lambda \forall \lambda, T>0
\end{aligned}
$$

Since by the Minkowski inequality,

$$
\operatorname{var}_{\mathbb{Q}}\left[\left|k\left(T \wedge \tau_{i}\right)-k^{*}\right|\right] \leq \mathbb{E}_{\mathbb{Q}}\left[\left|k\left(T \wedge \tau_{i}\right)-k^{*}\right|^{2}\right]-\left(k-k^{*}\right)^{2} \forall T>0
$$

Hence, by (D.2) we get

$$
\mathbb{E}_{\mathbb{Q}}\left[\left|k\left(T \wedge \tau_{i}\right)-k^{*}\right|^{2}\right] \leq\left(k+k^{*}\right) \lambda+\left(k-k^{*}\right)^{2}<\infty \forall \lambda, T>0
$$

Thus, $k\left(T \wedge \tau_{i}\right)-k^{*}(\forall T>0)$ is square-integrable martingale. Define

$$
\xi_{i} \triangleq\left|k\left(t \wedge \tau_{i}\right)-k^{*}\right| \quad \forall i \in \mathbb{N}
$$


And let

$$
\begin{aligned}
& \xi_{i}^{*} \triangleq\left\|k\left(t \wedge \tau_{i}\right)-k^{*}\right\|_{\infty} \triangleq \sup _{0 \leq s \leq t}\left|k\left(s \wedge \tau_{i}\right)-k^{*}\right| \quad \forall i \in \mathbb{N} \\
& \left\|k\left(t \wedge \tau_{i}\right)-k^{*}\right\|_{2} \triangleq\left\{\mathbb{E}_{\mathbb{Q}}\left[\left|k\left(t \wedge \tau_{i}\right)-k^{*}\right|^{2}\right]\right\}^{1 / 2} \quad \forall i \in \mathbb{N}
\end{aligned}
$$

denote the $L^{\infty}$-norm and $L^{2}$-norm, respectively. Let $\zeta>0$ be some constant, then by Doob's martingale inequality and Fubini theorem, we have

$$
\begin{aligned}
\mathbb{E}_{\mathbb{Q}}\left[\left|\xi_{i}^{*} \wedge \zeta\right|^{2}\right] & =2 \int_{0}^{\infty} \lambda \mathbb{Q}\left\{\omega ; \xi_{i}^{*}(\omega) \wedge \zeta \geq \lambda\right\} d \lambda \\
& \leq 2 \int_{0}^{\infty}\left(\int_{\left\{\omega ; \xi_{i}^{*}(\omega) \wedge \zeta \geq \lambda\right\}} \xi_{i}(\omega) d \mathbb{Q}(\omega)\right) d \lambda \\
& \left.=2 \int_{0}^{\infty}\left(\int_{\Omega} \xi_{i}(\omega) \chi_{\left\{\omega ; \xi_{i}^{*}\right.}(\omega) \wedge \zeta \geq \lambda\right\} d \mathbb{Q}(\omega)\right) d \lambda \\
& =2 \int_{\Omega} \xi_{i}(\omega)\left(\int_{0}^{\xi_{i}^{*} \wedge \zeta} d \lambda\right) d \mathbb{Q}(\omega) \\
& =2 \int_{\Omega} \xi_{i}(\omega)\left(\xi_{i}^{*}(\omega) \wedge \zeta\right) d \mathbb{Q}(\omega) \\
& =2 \mathbb{E}_{\mathbb{Q}}\left[\xi_{i}\left(\xi_{i}^{*} \wedge \zeta\right)\right]
\end{aligned}
$$

It follows from Hölder inequality and $\mathbb{E}_{\mathbb{Q}}\left[\left(\xi_{i}^{*} \wedge \zeta\right)^{2}\right] \leq \zeta^{2}<\infty$ that,

$$
\begin{aligned}
\left\|\xi_{i}^{*} \wedge \zeta\right\|_{2}^{2}=\mathbb{E}_{\mathbb{Q}}\left[\left(\xi_{i}^{*} \wedge \zeta\right)^{2}\right] & \leq 2\left\|\xi_{i}\right\|_{2}\left\|\xi_{i}^{*} \wedge \zeta\right\|_{2} \\
\Leftrightarrow\left\|\xi_{i}^{*} \wedge \zeta\right\|_{2} & \leq 2\left\|\xi_{i}\right\|_{2}
\end{aligned}
$$

Hence, applying Lebesgue dominated convergence theorem,

$$
\left\|\xi_{i}^{*}\right\|_{2}=\lim _{\zeta \rightarrow \infty}\left\|\xi_{i}^{*} \wedge \zeta\right\|_{2} \leq 2\left\|\xi_{i}\right\|_{2}
$$

i.e.,

$$
\begin{aligned}
\left\{\mathbb{E}_{\mathbb{Q}}\left[\sup _{0 \leq s \leq t}\left|k\left(s \wedge \tau_{i}\right)-k^{*}\right|^{2}\right]\right\}^{1 / 2} & \leq 2\left\{\mathbb{E}_{\mathbb{Q}}\left[\left|k\left(t \wedge \tau_{i}\right)-k^{*}\right|^{2}\right]\right\}^{1 / 2} \forall i \in \mathbb{N} \\
\Leftrightarrow \mathbb{E}_{\mathbb{Q}}\left[\sup _{0 \leq s \leq t}\left|k\left(s \wedge \tau_{i}\right)-k^{*}\right|^{2}\right] & \leq 4 \mathbb{E}_{\mathbb{Q}}\left[\left|k\left(t \wedge \tau_{i}\right)-k^{*}\right|^{2}\right] \\
& \leq 4 \lambda\left(k+k^{*}\right)+4\left(k-k^{*}\right)^{2} \forall \lambda>0, \forall i \in \mathbb{N}
\end{aligned}
$$


by (D.3). Letting $i \rightarrow \infty$ and by Lebesgue bounded convergence theorem,

$$
\mathbb{E}_{\mathbb{Q}}\left[\sup _{0 \leq s \leq t}\left|k(s)-k^{*}\right|^{2}\right] \leq 4 \lambda\left(k+k^{*}\right)+4\left(k-k^{*}\right)^{2} \forall \lambda>0
$$

Thus

$$
\lim _{t \rightarrow \infty} \sup _{0 \leq s \leq t}\left|k(s)-k^{*}\right|^{2}<\infty \text { a.s. }
$$

Therefore, there exists some constant $F$ such that

$$
\left|k(t)-k^{*}\right|^{2} \leq F \quad \forall t \geq 0
$$

almost surely. Moreover, since on the probability space $\left(\Omega, \mathcal{F}_{T}, \mathbb{Q}\right)$ we have

$$
d k(t)=-\sigma k(t) d \hat{B}(t)
$$

Hence, we can define the following characteristic operator of $k(t)$,

$$
\hat{\mathcal{A}} g(k) \triangleq \frac{1}{2} \sigma^{2} k^{2} \frac{\partial^{2} g}{\partial k^{2}}
$$

for any $k>0$. We define the Kullback-Leibler distance (see, Bomze, 1991; Imhof, 2005) between $k$ and $k^{*}$ as follows

$$
g(k) \triangleq \operatorname{dist}\left(k, k^{*}\right) \triangleq k^{*} \log \left(k^{*} / k\right) \geq 0
$$

Then,

$$
\hat{\mathcal{A}} g(k)=\frac{1}{2} \sigma^{2} k^{*}, \text { for any } k>0
$$

Thus, by (D.4),

$$
\hat{\mathcal{A}} g(k) \leq \frac{1}{2} \sigma^{2} k^{*}+2 F-\left|k-k^{*}\right|^{2} \triangleq-\left|k-k^{*}\right|^{2}+E
$$

where $E \triangleq\left(\sigma^{2} k^{*} / 2\right)+2 F>0$ is some constant. Define,

$$
\begin{aligned}
B_{\alpha}\left(k^{*}\right) & \triangleq\left\{k(t)>0 ;\left|k(t)-k^{*}\right|<\alpha, t \geq 0\right\} \\
\tilde{\tau} & \triangleq \tau_{\bar{B}_{\alpha}\left(k^{*}\right)} \triangleq \inf \left\{t ; k(t) \in \bar{B}_{\alpha}\left(k^{*}\right)\right\}
\end{aligned}
$$

where $\bar{B}_{\alpha}\left(k^{*}\right)$ denotes $B_{\alpha}\left(k^{*}\right)$ 's closure. Suppose that $\alpha^{2}>E$, for every $k \notin \bar{B}_{\alpha}\left(k^{*}\right)$, i.e., $k \in \bar{B}_{\alpha}^{C}\left(k^{*}\right)$, we have

$$
\hat{\mathcal{A}} g(k) \leq-\alpha^{2}+E
$$


by (D.5). Then by Dynkin's formula,

$$
\begin{aligned}
0 & \leq \mathbb{E}_{\mathbb{Q}}^{k}\{g[k(t \wedge \tilde{\tau})]\}=g(k)+\mathbb{E}_{\mathbb{Q}}^{k} \int_{0}^{t \wedge \tilde{\tau}} \hat{\mathcal{A}} g(k(s)) d s \\
& \leq g(k)+\left(E-\alpha^{2}\right) \mathbb{E}_{\mathbb{Q}}^{k}\{t \wedge \tilde{\tau}\}
\end{aligned}
$$

Since $t \wedge \tilde{\tau} \downarrow \tilde{\tau}$ as $t \rightarrow \infty$. Then by Lebesgue monotone convergence theorem, we have,

$$
0 \leq g(k)+\left(E-\alpha^{2}\right) \mathbb{E}_{\mathbb{Q}}^{k}\{\tilde{\tau}(\omega)\}
$$

which yields,

$$
\mathbb{E}_{\mathbb{Q}}^{k}\left[\tau_{\bar{B}_{\alpha}\left(k^{*}\right)}(\omega)\right]=\mathbb{E}_{\mathbb{Q}}^{k}[\tilde{\tau}(\omega)] \leq \frac{g(k)}{\alpha^{2}-E}=\frac{\operatorname{dist}\left(k, k^{*}\right)}{\alpha^{2}-E}
$$

as required in (i). Furthermore, for some constant $W>g(k)$, set up

$$
\tau_{W} \triangleq \inf \{t \geq 0 ; g(k(t))=W\}
$$

Then, by Dynkin's formula and inequality (D.5),

$$
\begin{aligned}
0 & \leq \mathbb{E}_{\mathbb{Q}}^{k}\left\{g\left[k\left(t \wedge \tau_{W}\right)\right]\right\}=g(k)+\mathbb{E}_{\mathbb{Q}}^{k} \int_{0}^{t \wedge \tau_{W}} \hat{\mathcal{A}} g(k(s)) d s \\
& \leq g(k)-\mathbb{E}_{\mathbb{Q}}^{k} \int_{0}^{t \wedge \tau_{W}}\left|k(s)-k^{*}\right|^{2} d s+E \mathbb{E}_{\mathbb{Q}}^{k}\left(t \wedge \tau_{W}\right)
\end{aligned}
$$

If $W \rightarrow \infty$, then $t \wedge \tau_{W} \rightarrow t$, and by Lebesgue bounded convergence theorem,

$$
0 \leq g(k)-\mathbb{E}_{\mathbb{Q}}^{k} \int_{0}^{t}\left|k(s)-k^{*}\right|^{2} d s+E t
$$

which yields,

$$
\mathbb{E}_{\mathbb{Q}}^{k}\left[\frac{1}{t} \int_{0}^{t}\left|k(s)-k^{*}\right|^{2} d s\right] \leq E
$$

Thus,

$$
\limsup _{t \rightarrow \infty} \mathbb{E}_{\mathbb{Q}}^{k}\left[\frac{1}{t} \int_{0}^{t}\left|k(s)-k^{*}\right|^{2} d s\right] \leq \frac{g(k)}{t}+E
$$

Then the required assertion in (ii) follows. If we let $\chi_{\bar{B}_{\alpha}^{C}\left(k^{*}\right)}$ denote the indicator function of $\bar{B}_{\alpha}^{C}\left(k^{*}\right)$, and let $\nu$, induced by Brownian motion 
$\hat{B}(t), t \geq 0$, denote the Wiener measure (see, Karatzas and Shreve, 1991, pp.71) on Borel sigma algebra $\mathfrak{B}(C[0, \infty))$ generated by $k(t), t \geq 0$, then we get

$$
\begin{aligned}
\nu\left[\bar{B}_{\alpha}^{C}\left(k^{*}\right)\right] & =\limsup _{t \rightarrow \infty} \mathbb{E}_{\mathbb{Q}}^{k}\left[\frac{1}{t} \int_{0}^{t} \chi_{\bar{B}_{\alpha}^{C}\left(k^{*}\right)}(k(s)) d s\right] \\
& \leq \limsup _{t \rightarrow \infty} \mathbb{E}_{\mathbb{Q}}^{k}\left[\frac{1}{t} \int_{0}^{t} \frac{\left|k(s)-k^{*}\right|^{2}}{\alpha^{2}} d s\right] \leq E / \alpha^{2}
\end{aligned}
$$

Hence we have,

$$
\nu\left[\bar{B}_{\alpha}\left(k^{*}\right)\right] \geq 1-\frac{E}{\alpha^{2}}
$$

which gives the desired result in (iii).

\section{APPENDIX E}

\section{Proof of Theorem 4}

By (6), we have

$$
d k(t)=f(k(t)) d t+g(k(t)) d B(t)
$$

where

$$
\begin{gathered}
f(k(t)) \triangleq\left[r^{s} A-\delta-(A-\delta) r^{t}+\sigma^{2}-n\right] k(t) \triangleq \varpi k(t) \\
g(k(t)) \triangleq-\sigma k(t)
\end{gathered}
$$

Now, by Itô formula,

$$
\begin{aligned}
\left|k(t)-k^{*}\right|^{2}= & \left|k(0)-k^{*}\right|^{2}+2 \int_{0}^{t}\left\langle k(s)-k^{*}, f(k(s))\right\rangle d s \\
& +2 \int_{0}^{t}\left\langle k(s)-k^{*}, g(k(s)) d B(s)\right\rangle+\int_{0}^{t}|g(k(s))|^{2} d s
\end{aligned}
$$

where $\langle\cdot, \cdot\rangle$ denotes the standard inner product. For $t_{1} \in[0, T]$, and $\eta=\eta(p)$, we get

$$
\begin{aligned}
\sup _{0 \leq t \leq t_{1}}\left|k(t)-k^{*}\right|^{p} & \leq \eta\left\{\left|k(0)-k^{*}\right|^{p}+\left[\int_{0}^{t_{1}}\left(\sigma^{2}|k(s)|^{2}\right) d s\right]^{p / 2}\right. \\
& +\sup _{0 \leq t \leq t_{1}}\left|\int_{0}^{t}\left\langle k(s)-k^{*}, f(k(s))\right\rangle d s\right|^{p / 2} \\
& \left.+\sup _{0 \leq t \leq t_{1}}\left|\int_{0}^{t}\left\langle k(s)-k^{*}, g(k(s)) d B(s)\right\rangle\right|^{p / 2}\right\}
\end{aligned}
$$


It follows from Cauchy-Schwarz inequality that

$$
\begin{aligned}
\sup _{0 \leq t \leq t_{1}}\left|k(t)-k^{*}\right|^{p} & \leq \eta\left\{\left|k(0)-k^{*}\right|^{p}+|\sigma|^{p} T^{(p-2) / 2} \int_{0}^{t_{1}}|k(s)|^{p} d s\right. \\
& +\sup _{0 \leq t \leq t_{1}}\left|\int_{0}^{t}\left\langle k(s)-k^{*}, f(k(s))\right\rangle d s\right|^{p / 2} \\
& \left.+\sup _{0 \leq t \leq t_{1}}\left|\int_{0}^{t}\left\langle k(s)-k^{*}, g(k(s)) d B(s)\right\rangle\right|^{p / 2}\right\}
\end{aligned}
$$

Taking expectations and for some $\underline{\eta}=\underline{\eta}(p)$, we have

$$
\begin{aligned}
\mathbb{E}\left[\sup _{0 \leq t \leq t_{1}}\left|k(t)-k^{*}\right|^{p}\right] & \leq|\sigma|^{p} T^{(p-2) / 2} \underline{\eta}\left\{\mathbb{E}\left|k(0)-k^{*}\right|^{p}+\mathbb{E}\left[\int_{0}^{t_{1}}|k(s)|^{p} d s\right]\right. \\
& +\mathbb{E}\left[\sup _{0 \leq t \leq t_{1}}\left|\int_{0}^{t}\left\langle k(s)-k^{*}, f(k(s))\right\rangle d s\right|^{p / 2}\right] \\
& \left.+\mathbb{E}\left[\sup _{0 \leq t \leq t_{1}}\left|\int_{0}^{t}\left\langle k(s)-k^{*}, g(k(s)) d B(s)\right\rangle\right|^{p / 2}\right]\right\}
\end{aligned}
$$

Applying Burkholder-Davis-Gundy inequality (see, Karatzas and Shreve, 1991, pp.166), for some $\tilde{\eta}=\tilde{\eta}(p)$

$$
\begin{aligned}
\mathbb{E}\left[\sup _{0 \leq t \leq t_{1}}\left|k(t)-k^{*}\right|^{p}\right] & \leq|\sigma|^{p} T^{(p-2) / 2} \tilde{\eta}\left\{\mathbb{E}\left|k(0)-k^{*}\right|^{p}+\int_{0}^{t_{1}} \mathbb{E}|k(s)|^{p} d s\right. \\
& +\mathbb{E}\left[\int_{0}^{t_{1}}\left|k(s)-k^{*}\right|^{2}|f(k(s))|^{2} d s\right]^{p / 4} \\
& \left.+\mathbb{E}\left[\int_{0}^{t_{1}}\left|k(s)-k^{*}\right|^{2}|g(k(s))|^{2} d s\right]^{p / 4}\right\}
\end{aligned}
$$

Next, by the Young inequality (see, Higham et al, 2003) and Hölder inequality,

$$
\begin{aligned}
& \mathbb{E}\left[\int_{0}^{t_{1}}\left|k(s)-k^{*}\right|^{2}|f(k(s))|^{2} d s\right]^{p / 4} \leq \mathbb{E}\left[\sup _{0 \leq s \leq t_{1}}\left|k(s)-k^{*}\right|^{p / 2}\left(\int_{0}^{t_{1}}|f(k(s))|^{2} d s\right)^{p / 4}\right] \\
\leq & \frac{1}{2\left(2|\sigma|^{p} T^{(p-2) / 2} \tilde{\eta}\right)} \mathbb{E}\left[\sup _{0 \leq s \leq t_{1}}\left|k(s)-k^{*}\right|^{p}\right]+\frac{\left(2|\sigma|^{p} T^{(p-2) / 2} \tilde{\eta}\right)}{2} \mathbb{E}\left[\int_{0}^{t_{1}}|f(k(s))|^{2} d s\right]^{p / 2} \\
\leq & \frac{1}{2\left(2|\sigma|^{p} T^{(p-2) / 2} \tilde{\eta}\right)} \mathbb{E}\left[\sup _{0 \leq s \leq t_{1}}\left|k(s)-k^{*}\right|^{p}\right] \\
+ & \frac{\left(2|\sigma|^{p} T^{(p-2) / 2} \tilde{\eta}\right)}{2} T^{(p-2) / 2}|\varpi|^{p} \mathbb{E}\left[\int_{0}^{t_{1}}|k(s)|^{p} d s\right]
\end{aligned}
$$


Similarly, we get

$$
\begin{aligned}
& \mathbb{E}\left[\int_{0}^{t_{1}}\left|k(s)-k^{*}\right|^{2}|g(k(s))|^{2} d s\right]^{p / 4} \\
\leq & \frac{1}{2\left(2|\sigma|^{p} T^{(p-2) / 2} \tilde{\eta}\right)} \mathbb{E}\left[\sup _{0 \leq s \leq t_{1}}\left|k(s)-k^{*}\right|^{p}\right] \\
+ & \frac{\left(2|\sigma|^{p} T^{(p-2) / 2} \tilde{\eta}\right)}{2} T^{(p-2) / 2}|\sigma|^{p} \mathbb{E}\left[\int_{0}^{t_{1}}|k(s)|^{p} d s\right]
\end{aligned}
$$

Hence, substituting (E.2) and (E.3) into (E.1) yields,

$$
\begin{gathered}
\mathbb{E}\left[\sup _{0 \leq t \leq t_{1}}\left|k(t)-k^{*}\right|^{p}\right] \leq 2|\sigma|^{p} T^{(p-2) / 2} \tilde{\eta}\left\{\mathbb{E}\left[\left|k(0)-k^{*}\right|^{p}\right]\right. \\
\left.+\int_{0}^{t_{1}} \mathbb{E}|k(s)|^{p} d s+\tilde{\eta} T^{p-2}|\sigma|^{p} \mathbb{E}\left[\int_{0}^{t_{1}}|k(s)|^{p} d s\right]\left(|\sigma|^{p}+|\varpi|^{p}\right)\right\}
\end{gathered}
$$

There must be some $\bar{\eta}=\bar{\eta}(p)$ such that,

$$
\begin{aligned}
& \mathbb{E}\left[\sup _{0 \leq t \leq t_{1}}\left|k(s)-k^{*}\right|^{p}\right] \\
\leq & 2|\sigma|^{2 p} T^{3(p-2) / 2}\left(|\sigma|^{p}+|\varpi|^{p}\right) \bar{\eta}(p)\left(\mathbb{E}\left|k(0)-k^{*}\right|^{p}+\int_{0}^{T} \mathbb{E}|k(s)|^{p} d s\right)
\end{aligned}
$$

Since by (6),

$$
k(t)=k(0) \exp \left\{\left[r^{s} A-\delta-(A-\delta) r^{t}+\left(\sigma^{2} / 2\right)-n\right] t-\sigma B(t)\right\}
$$

Thus

$$
\begin{aligned}
\mathbb{E}\left[|k(t)|^{p}\right] & =|k(0)|^{p} \mathbb{E}\left[\exp \left\{p\left[r^{s} A-\delta-(A-\delta) r^{t}+\left(\sigma^{2} / 2\right)-n\right] t-p \sigma B(t)\right\}\right] \\
& =|k(0)|^{p} \exp (\widehat{\eta}(p) t)
\end{aligned}
$$

where

$$
\widehat{\eta}(p) \triangleq p\left[r^{s} A-\delta-(A-\delta) r^{t}+\left(\sigma^{2} / 2\right)-n\right]+\left(p^{2} \sigma^{2} / 2\right)
$$

Hence

$$
\int_{0}^{T} \mathbb{E}\left[|k(t)|^{p}\right] d s=\frac{|k(0)|^{p}}{\widehat{\eta}}[\exp (\widehat{\eta} T)-1]
$$


Therefore,

$$
\begin{aligned}
& \mathbb{E}\left[\sup _{0 \leq t \leq T}\left|k(t)-k^{*}\right|^{p}\right] \\
\leq & 2|\sigma|^{2 p} T^{3(p-2) / 2}\left(|\sigma|^{p}+|\varpi|^{p}\right) \bar{\eta}(p)\left(\left|k(0)-k^{*}\right|^{p}+\frac{|k(0)|^{p}}{\widehat{\eta}}[\exp (\widehat{\eta} T)-1]\right)
\end{aligned}
$$

Put

$C(p, T) \triangleq 2|\sigma|^{2 p} T^{3(p-2) / 2}\left(|\sigma|^{p}+|\varpi|^{p}\right) \bar{\eta}(p)\left(\left|k(0)-k^{*}\right|^{p}+\frac{|k(0)|^{p}}{\widehat{\eta}}[\exp (\widehat{\eta} T)-1]\right)$

Then we have

$$
\mathbb{E}\left[\sup _{0 \leq t \leq T}\left|k(t)-k^{*}\right|^{p}\right] \leq C(p, T)
$$

In particular, when $\sigma \rightarrow 0$, by Levi lemma we have

$$
\lim _{T \rightarrow \infty} \mathbb{E}\left[\sup _{0 \leq t \leq T}\left|k(t)-k^{*}\right|^{p}\right]=\mathbb{E}\left[\lim _{T \rightarrow \infty} \sup _{0 \leq t \leq T}\left|k(t)-k^{*}\right|^{p}\right] \rightarrow 0
$$

\section{Proof of Theorem 5}

\section{APPENDIX F}

Noting that

$$
\mathbb{E}\left[\sup _{0 \leq t \leq T}\left|\bar{c}^{*}(t)-\tilde{c}^{*}(t)\right|^{2}\right]=\rho \mathbb{E}\left[\sup _{0 \leq t \leq T}|\bar{k}(t)-\tilde{k}(t)|^{2}\right]
$$

Hence, we now prove that

$$
\mathbb{E}\left[\lim _{T \rightarrow \infty} \sup _{0 \leq t \leq T}|\bar{k}(t)-\tilde{k}(t)|^{2}\right] \rightarrow 0
$$

From Lemma 1 and for $\forall 2<p<\infty$ there is some constant $W$ such that

$$
\mathbb{E}\left[\sup _{0 \leq t \leq T}|\bar{k}(t)|^{p}\right] \vee \mathbb{E}\left[\sup _{0 \leq t \leq T}|\tilde{k}(t)|^{p}\right] \leq W
$$

where

$$
\begin{aligned}
& \bar{k}(t)=k(0)+\int_{0}^{t}\left[r^{s} A-\delta-(A-\delta) \bar{r}_{0}^{t}+\sigma^{2}-n\right] \bar{k}(s) d s+\int_{0}^{t}(-\sigma) \bar{k}(s) d B(s) \\
& \tilde{k}(t)=k(0)+\int_{0}^{t}\left[r^{s} A-\delta-(A-\delta) \tilde{r}_{0}^{t}+\sigma^{2}-n\right] \tilde{k}(s) d s+\int_{0}^{t}(-\sigma) \tilde{k}(s) d B(s)
\end{aligned}
$$


Suppose $|\bar{k}(t)| \vee|\tilde{k}(t)| \leq \bar{W}, \forall t \geq 0$, otherwise we just consider $\bar{k}(t) \wedge \bar{W}$ and $\tilde{k}(t) \wedge \bar{W}$ instead of $\bar{k}(t)$ and $\tilde{k}(t)$, respectively, for some $0 \leq \bar{W}<\infty$. In what follows, we firstly define the following stopping time,

$$
\bar{\tau}_{\bar{W}} \triangleq \inf \{t \geq 0 ;|\bar{k}(t)| \geq \bar{W}\}, \tilde{\tau}_{\bar{W}} \triangleq \inf \{t \geq 0 ;|\tilde{k}(t)| \geq \bar{W}\}, \tau_{\bar{W}} \triangleq \bar{\tau}_{\bar{W}} \wedge \tilde{\tau}_{\bar{W}}
$$

By the Young inequality (see, Higham et al, 2003) and for any $v>0$,

$$
\begin{aligned}
& \mathbb{E}\left[\sup _{0 \leq t \leq T}|\bar{k}(t)-\tilde{k}(t)|^{2}\right] \\
= & \mathbb{E}\left[\sup _{0 \leq t \leq T}|\bar{k}(t)-\tilde{k}(t)|^{2} \chi_{\left\{\bar{\tau}_{\bar{W}}>T, \tilde{\tau}_{\bar{W}}>T\right\}}\right] \\
+ & \mathbb{E}\left[\sup _{0 \leq t \leq T}|\bar{k}(t)-\tilde{k}(t)|^{2} \chi_{\left\{\bar{\tau}_{\bar{W}} \leq T, \text { or } \tilde{\tau}_{\bar{W}} \leq T\right\}}\right] \\
\leq & \mathbb{E}\left[\sup _{0 \leq t \leq T}\left|\bar{k}\left(t \wedge \tau_{\bar{W}}\right)-\tilde{k}\left(t \wedge \tau_{\bar{W}}\right)\right|^{2} \chi_{\left\{\tau_{\bar{W}}>T\right\}}\right] \\
+ & \frac{2 v}{p} \mathbb{E}\left[\sup _{0 \leq t \leq T}|\bar{k}(t)-\tilde{k}(t)|^{p}\right]+\frac{1-(2 / p)}{v^{2 /(p-2)}} \mathbb{P}\left\{\bar{\tau}_{\bar{W}} \leq T, \text { or } \tilde{\tau}_{\bar{W}} \leq T\right\}
\end{aligned}
$$

It follows from (F.2) that,

$$
\mathbb{P}\left\{\bar{\tau}_{\bar{W}} \leq T\right\}=\mathbb{E}\left[\chi_{\left\{\bar{\tau}_{\bar{W}} \leq T\right\}} \frac{\bar{k}(\overline{\bar{\tau}} \bar{W})^{p}}{\bar{W}^{p}}\right] \leq \frac{1}{\bar{W}^{p}} \mathbb{E}\left[\sup _{0 \leq t \leq T}|\bar{k}(t)|^{p}\right] \leq \frac{W}{\bar{W}^{p}}
$$

And similarly, $\mathbb{P}\left\{\tilde{\tau}_{\bar{W}} \leq T\right\} \leq\left(W / \bar{W}^{p}\right)$. So,

$$
\mathbb{P}\left\{\bar{\tau}_{\bar{W}} \leq T, \text { or } \tilde{\tau}_{\bar{W}} \leq T\right\} \leq \mathbb{P}\left\{\bar{\tau}_{\bar{W}} \leq T\right\}+\mathbb{P}\left\{\tilde{\tau}_{\bar{W}} \leq T\right\} \leq \frac{2 W}{\bar{W}^{p}}
$$

Thus we obtain,

$$
\mathbb{E}\left[\sup _{0 \leq t \leq T}|\bar{k}(t)-\tilde{k}(t)|^{p}\right] \leq 2^{p-1} \mathbb{E}\left[\sup _{0 \leq t \leq T}\left(|\bar{k}(t)|^{p}+|\tilde{k}(t)|^{p}\right)\right] \leq 2^{p} W
$$

Hence (F.3) becomes,

$$
\begin{aligned}
\mathbb{E}\left[\sup _{0 \leq t \leq T}|\bar{k}(t)-\tilde{k}(t)|^{2}\right] & \leq \mathbb{E}\left[\sup _{0 \leq t \leq T}\left|\bar{k}\left(t \wedge \tau_{\bar{W}}\right)-\tilde{k}\left(t \wedge \tau_{\bar{W}}\right)\right|^{2}\right] \\
& +\frac{2^{p+1} v W}{p}+\frac{(p-2) 2 W}{p v^{2 / p-2} \bar{W}^{p}}
\end{aligned}
$$


Define

$$
\begin{aligned}
& \bar{\kappa} \triangleq r^{s} A-\delta-(A-\delta) \bar{r}_{0}^{t}+\sigma^{2}-n \\
& \tilde{\kappa} \triangleq r^{s} A-\delta-(A-\delta) \tilde{r}_{0}^{t}+\sigma^{2}-n
\end{aligned}
$$

Thus by the Cauchy-Schwarz inequality and the triangle inequality,

$$
\begin{aligned}
& \left|\bar{k}\left(t \wedge \tau_{\bar{W}}\right)-\tilde{k}\left(t \wedge \tau_{\bar{W}}\right)\right|^{2} \\
= & \left|\int_{0}^{t \wedge \tau_{\bar{W}}}[\bar{\kappa} \bar{k}(s)-\tilde{\kappa} \tilde{k}(s)] d s+\int_{0}^{t \wedge \tau_{\bar{W}}} \sigma[\tilde{k}(s)-\bar{k}(s)] d B(s)\right|^{2} \\
\leq & 2\left[T \int_{0}^{t \wedge \tau_{\bar{W}}}|\bar{\kappa} \bar{k}(s)-\tilde{\kappa} \tilde{k}(s)|^{2} d s+\sigma^{2}\left|\int_{0}^{t \wedge \tau_{\bar{W}}}[\tilde{k}(s)-\bar{k}(s)] d B(s)\right|^{2}\right] \\
\leq & 2 T \int_{0}^{t \wedge \tau_{\bar{W}}}|\bar{\kappa}|^{2}|\bar{k}(s)-\tilde{k}(s)|^{2} d s+2 T \int_{0}^{t \wedge \tau_{\bar{W}}}|\bar{\kappa}-\tilde{\kappa}|^{2}|\tilde{k}(s)|^{2} d s \\
& +2 \sigma^{2}\left|\int_{0}^{t \wedge \tau_{\bar{W}}}[\tilde{k}(s)-\bar{k}(s)] d B(s)\right|^{2}
\end{aligned}
$$

So for any $\tau \leq T$, by Itô isometry, we have

$$
\begin{aligned}
& \mathbb{E}\left[\sup _{0 \leq t \leq \tau} \mid \bar{k}\left(t \wedge \tau_{\bar{W}}\right)-\tilde{k}\left(\left.t \wedge \tau_{\bar{W}}\right|^{2}\right]\right. \\
\leq & 2\left(T|\bar{\kappa}|^{2}+\sigma^{2}\right) \mathbb{E}\left[\int_{0}^{t \wedge \tau_{\bar{W}}}|\bar{k}(s)-\tilde{k}(s)|^{2} d s\right]+2 T|\bar{\kappa}-\tilde{\kappa}|^{2} \int_{0}^{T} \mathbb{E}|\tilde{k}(s)|^{2} d s \\
\leq & 2\left(T|\bar{\kappa}|^{2}+\sigma^{2}\right) \int_{0}^{T} \mathbb{E}\left[\sup _{0 \leq t_{0} \leq s}\left|\bar{k}\left(t_{0} \wedge \tau_{\bar{W}}\right)-\tilde{k}\left(t_{0} \wedge \tau_{\bar{W}}\right)\right|^{2}\right] d s \\
& +2 T|\bar{\kappa}-\tilde{\kappa}|^{2} \int_{0}^{T} \mathbb{E}|\tilde{k}(s)|^{2} d s
\end{aligned}
$$

Since by (6),

$$
\tilde{k}(t)=k(0) \exp \left\{\left[r^{s} A-\delta-(A-\delta) \tilde{r}_{0}^{t}+\left(\sigma^{2} / 2\right)-n\right] t-\sigma B(t)\right\}
$$

Thus

$$
\mathbb{E}\left[|\tilde{k}(t)|^{2}\right]=|k(0)|^{2} \exp \left(\tilde{\kappa}_{1} t\right)
$$

Where

$$
\tilde{\kappa} \triangleq 2 r^{s} A-2 \delta-2(A-\delta) \tilde{r}_{0}^{t}+3 \sigma^{2}-2 n
$$

Then

$$
\int_{0}^{T} \mathbb{E}|\tilde{k}(t)|^{2} d t=\frac{|k(0)|^{2}}{\tilde{\kappa}_{1}}\left(\exp \left(\tilde{\kappa}_{1} T\right)-1\right)
$$


Accordingly,

$$
\begin{aligned}
& \mathbb{E}\left[\sup _{0 \leq t \leq \tau}\left|\bar{k}\left(t \wedge \tau_{\bar{W}}\right)-\tilde{k}\left(t \wedge \tau_{\bar{W}}\right)\right|^{2}\right] \\
\leq & 2\left(T|\bar{\kappa}|^{2}+\sigma^{2}\right) \int_{0}^{\tau} \mathbb{E}\left[\sup _{0 \leq t_{0} \leq s}\left|\bar{k}\left(t_{0} \wedge \tau_{\bar{W}}\right)-\tilde{k}\left(t_{0} \wedge \tau_{\bar{W}}\right)\right|^{2}\right] d s \\
& +2 T|\bar{\kappa}-\tilde{\kappa}|^{2}\left(|k(0)|^{2} / \tilde{\kappa}_{1}\right)\left(\exp \left(\tilde{\kappa}_{1} T\right)-1\right)
\end{aligned}
$$

So the Gronwall's inequality (see, Higham et al, 2003) yields

$$
\begin{aligned}
& \mathbb{E}\left[\sup _{0 \leq t \leq \tau}\left|\bar{k}\left(t \wedge \tau_{\bar{W}}\right)-\tilde{k}\left(t \wedge \tau_{\bar{W}}\right)\right|^{2}\right] \\
\leq & 2 T|\bar{\kappa}-\tilde{\kappa}|^{2} \frac{|k(0)|^{2}}{\tilde{\kappa}_{1}}\left(\exp \left(\tilde{\kappa}_{1} T\right)-1\right) \exp \left[2\left(T|\bar{\kappa}|^{2}+\sigma^{2}\right) T\right]
\end{aligned}
$$

Inserting this into (F.4) gives

$$
\begin{aligned}
& \mathbb{E}\left[\sup _{0 \leq t \leq T}|\bar{k}(t)-\tilde{k}(t)|^{2}\right] \\
\leq & 2 T|\bar{\kappa}-\tilde{\kappa}|^{2} \frac{|k(0)|^{2}}{\tilde{\kappa}_{1}}\left(\exp \left(\tilde{\kappa}_{1} T\right)-1\right) e^{2\left(T|\bar{\kappa}|^{2}+\sigma^{2}\right) T} \\
& +\frac{2^{p+1} v W}{p}+\frac{2(p-2) W}{p v^{2 /(p-2)} \bar{W}^{p}}
\end{aligned}
$$

Hence, for $\forall \varepsilon>0$, we can choose some $v$ and $\bar{W}$ such that,

$$
\frac{2^{p+1} v W}{p} \leq \frac{\varepsilon}{3} \text { and } \frac{2(p-2) W}{p v^{2 /(p-2)} \bar{W}^{p}} \leq \frac{\varepsilon}{3}
$$

And for any given $T$, we put $k(0)$ such that

$$
2 T|\bar{\kappa}-\tilde{\kappa}|^{2} \frac{|k(0)|^{2}}{\tilde{\kappa}_{1}}\left(\exp \left(\tilde{\kappa}_{1} T\right)-1\right) e^{2\left(T|\bar{\kappa}|^{2}+\sigma^{2}\right) T} \leq \varepsilon / 3
$$

So, for $\forall \varepsilon>0$,

$$
\mathbb{E}\left[\sup _{0 \leq t \leq T}|\bar{k}(t)-\tilde{k}(t)|^{2}\right] \leq \varepsilon
$$

Therefore, we have

$$
\lim _{T \rightarrow \infty} \mathbb{E}\left[\sup _{0 \leq t \leq T}|\bar{k}(t)-\tilde{k}(t)|^{2}\right] \rightarrow 0, \text { as } \varepsilon \rightarrow 0
$$


By Levi lemma, we obtain

$$
\mathbb{E}\left[\lim _{T \rightarrow \infty} \sup _{0 \leq t \leq T}|\bar{k}(t)-\tilde{k}(t)|^{2}\right] \rightarrow 0
$$

which yields,

$$
\mathbb{E}\left[\lim _{T \rightarrow \infty} \sup _{0 \leq t \leq T}\left|\bar{c}^{*}(t)-\tilde{c}^{*}(t)\right|^{2}\right] \rightarrow 0 \text { as } \varepsilon \rightarrow 0
$$

by (F.1).

\section{APPENDIX G}

\section{Proof of Theorem 6}

By (23), we have,

$$
d c^{*}(t)=f(k(t)) d t+g(k(t)) d B(t)
$$

Where

$$
\begin{gathered}
f(k(t)) \triangleq \rho\left[r^{s} A-\delta-(A-\delta) r^{t}+\sigma^{2}-n\right] k(t) \triangle \bar{\omega} k(t) \\
g(k(t)) \triangleq-\sigma \rho k(t)
\end{gathered}
$$

Now, by Itô formula,

$$
\begin{aligned}
\left|c^{*}(t)-c^{*}\right|^{2}= & \left|c^{*}(0)-c^{*}\right|^{2}+2 \int_{0}^{t}\left\langle c^{*}(s)-c^{*}, f(k(s))\right\rangle d s \\
& +2 \int_{0}^{t}\left\langle c^{*}(s)-c^{*}, g(k(s)) d B(s)\right\rangle+\int_{0}^{t}|g(k(s))|^{2} d s
\end{aligned}
$$

where $\langle\cdot, \cdot\rangle$ denotes the standard inner product. For $t_{1} \in[0, T]$, and $\zeta=\zeta(p), \forall p \geq 2$ we get

$$
\begin{aligned}
\sup _{0 \leq t \leq t_{1}}\left|c^{*}(t)-c^{*}\right|^{p} & \leq \zeta\left\{\left|c^{*}(0)-c^{*}\right|^{p}+\left[\int_{0}^{t_{1}}\left(\rho^{2} \sigma^{2}|k(s)|^{2}\right) d s\right]^{p / 2}\right. \\
& +\sup _{0 \leq t \leq t_{1}}\left|\int_{0}^{t}\left\langle c^{*}(s)-c^{*}, f(k(s))\right\rangle d s\right|^{p / 2} \\
& \left.+\sup _{0 \leq t \leq t_{1}}\left|\int_{0}^{t}\left\langle c^{*}(s)-c^{*}, g(k(s)) d B(s)\right\rangle\right|^{p / 2}\right\}
\end{aligned}
$$


It follows from Cauchy-Schwarz inequality that

$$
\begin{aligned}
\sup _{0 \leq t \leq t_{1}}\left|c^{*}(t)-c^{*}\right|^{p} & \leq \zeta\left\{\left|c^{*}(0)-c^{*}\right|^{p}+|\rho \sigma|^{p} T^{(p-2) / 2} \int_{0}^{t_{1}}|k(s)|^{p} d s\right. \\
& +\sup _{0 \leq t \leq t_{1}}\left|\int_{0}^{t}\left\langle c^{*}(s)-c^{*}, f(k(s))\right\rangle d s\right|^{p / 2} \\
& \left.+\sup _{0 \leq t \leq t_{1}}\left|\int_{0}^{t}\left\langle c^{*}(s)-c^{*}, g(k(s)) d B(s)\right\rangle\right|^{p / 2}\right\}
\end{aligned}
$$

Taking expectations and for some $\underline{\zeta}=\underline{\zeta}(p)$, we have

$$
\begin{aligned}
\mathbb{E}\left[\sup _{0 \leq t \leq t_{1}}\left|c^{*}(t)-c^{*}\right|^{p}\right] & \leq|\rho \sigma|^{p} T^{(p-2) / 2} \underline{\zeta}\left\{\mathbb{E}\left|c^{*}(0)-c^{*}\right|^{p}+\mathbb{E}\left[\int_{0}^{t_{1}}|k(s)|^{p} d s\right]\right. \\
& +\mathbb{E}\left[\sup _{0 \leq t \leq t_{1}}\left|\int_{0}^{t}\left\langle c^{*}(s)-c^{*}, f(k(s))\right\rangle d s\right|^{p / 2}\right] \\
& \left.+\mathbb{E}\left[\sup _{0 \leq t \leq t_{1}}\left|\int_{0}^{t}\left\langle c^{*}(s)-c^{*}, g(k(s)) d B(s)\right\rangle\right|^{p / 2}\right]\right\}
\end{aligned}
$$

Applying Burkholder-Davis-Gundy inequality (see, Karatzas and Shreve, 1991, pp.166), for some $\tilde{\zeta}=\tilde{\zeta}(p)$,

$$
\begin{aligned}
\mathbb{E}\left[\sup _{0 \leq t \leq t_{1}}\left|c^{*}(t)-c^{*}\right|^{p}\right] & \leq|\rho \sigma|^{p} T^{(p-2) / 2} \tilde{\zeta}\left\{\mathbb{E}\left|c^{*}(0)-c^{*}\right|^{p}+\int_{0}^{t_{1}} \mathbb{E}|k(s)|^{p} d s\right. \\
& +\mathbb{E}\left[\int_{0}^{t_{1}}\left|c^{*}(s)-c^{*}\right|^{2}|f(k(s))|^{2} d s\right]^{p / 4} \\
& \left.+\mathbb{E}\left[\int_{0}^{t_{1}}\left|c^{*}(s)-c^{*}\right|^{2}|g(k(s))|^{2} d s\right]^{p / 4}\right\}
\end{aligned}
$$

Next, by the Young inequality (see, Higham et al, 2003) and Hölder inequality,

$$
\begin{aligned}
& \mathbb{E}\left[\int_{0}^{t_{1}}\left|c^{*}(s)-c^{*}\right|^{2}|f(k(s))|^{2} d s\right]^{p / 4} \leq \mathbb{E}\left[\sup _{0 \leq s \leq t_{1}}\left|c^{*}(s)-c^{*}\right|^{p / 2}\left(\int_{0}^{t_{1}}|f(k(s))|^{2} d s\right)^{p / 4}\right] \\
\leq & \frac{1}{2\left(2|\rho \sigma|^{p} T^{(p-2) / 2} \tilde{\zeta}\right)} \mathbb{E}\left[\sup _{0 \leq s \leq t_{1}}\left|c^{*}(s)-c^{*}\right|^{p}\right]+\frac{\left(2|\rho \sigma|^{p} T^{(p-2) / 2} \tilde{\zeta}\right)}{2} \mathbb{E}\left[\int_{0}^{t_{1}}|f(k(s))|^{2} d s\right]^{p / 2}(\mathrm{G} .2) \\
\leq & \frac{1}{2\left(2|\rho \sigma|^{p} T^{p-2) / 2} \tilde{\zeta}\right)} \mathbb{E}\left[\sup _{0 \leq s \leq t_{1}}\left|c^{*}(s)-c^{*}\right|^{p}\right]+\frac{\left(2|\rho \sigma|^{p} T^{(p-2) / 2} \tilde{\zeta}\right)}{2} T^{(p-2) / 2}|\bar{\omega}|^{p} \mathbb{E}\left[\int_{0}^{t_{1}}|k(s)|^{p} d s\right]
\end{aligned}
$$


Similarly, we get

$$
\begin{aligned}
& \mathbb{E}\left[\int_{0}^{t_{1}}\left|c^{*}(s)-c^{*}\right|^{2}|g(k(s))|^{2} d s\right]^{p / 4} \\
\leq & \frac{1}{2\left(2|\rho \sigma|^{p} T^{(p-2) / 2} \tilde{\zeta}\right)} \mathbb{E}\left[\sup _{0 \leq s \leq t_{1}}\left|c^{*}(s)-c^{*}\right|^{p}\right] \\
+ & \frac{\left(2|\rho \sigma|^{p} T^{(p-2) / 2} \tilde{\zeta}\right)}{2} T^{(p-2) / 2}|\rho \sigma|^{p} \mathbb{E}\left[\int_{0}^{t_{1}}|k(s)|^{p} d s\right]
\end{aligned}
$$

Hence, substituting (G.2) and (G.3) into (G.1) yields,

$$
\begin{gathered}
\mathbb{E}\left[\sup _{0 \leq t \leq t_{1}}\left|c^{*}(t)-c^{*}\right|^{p}\right] \leq 2|\rho \sigma|^{p} T^{(p-2) / 2} \tilde{\zeta}\left\{\mathbb{E}\left[\left|c^{*}(0)-c^{*}\right|^{p}\right]\right. \\
\left.+\int_{0}^{t_{1}} \mathbb{E}|k(s)|^{p} d s+\tilde{\zeta} T^{p-2} \mathbb{E}\left[\int_{0}^{t_{1}}|k(s)|^{p} d s\right]\left(|\rho \sigma|^{2 p}+|\rho \sigma|^{p}|\bar{\omega}|^{p}\right)\right\}
\end{gathered}
$$

Then there must be some $\bar{\zeta}=\bar{\zeta}(p)$ such that,

$$
\begin{aligned}
& \mathbb{E}\left[\sup _{0 \leq t \leq t_{1}}\left|c^{*}(t)-c^{*}\right|^{p}\right] \\
\leq & 2|\rho \sigma|^{2 p} T^{3(p-2) / 2}\left(|\rho \sigma|^{p}+|\bar{\omega}|^{p}\right) \bar{\zeta}(p)\left(\mathbb{E}\left|c^{*}(0)-c^{*}\right|^{p}+\int_{0}^{T} \mathbb{E}|k(s)|^{p} d s\right)
\end{aligned}
$$

Since by (6),

$$
k(t)=k(0) \exp \left\{\left[r^{s} A-\delta-(A-\delta) r^{t}+\left(\sigma^{2} / 2\right)-n\right] t-\sigma B(t)\right\}
$$

Thus

$$
\begin{aligned}
\left.\mathbb{E} \mid k(t)^{p}\right] & =|k(0)|^{p} \mathbb{E}\left[\exp \left\{p\left[r^{s} A-\delta-(A-\delta) r^{t}+\left(\sigma^{2} / 2\right)-n\right] t-p \sigma B(t)\right\}\right] \\
& =|k(0)|^{p} \exp (\widehat{\zeta}(p) t)
\end{aligned}
$$

where

$$
\widehat{\zeta}(p) \triangleq p\left[r^{s} A-\delta-(A-\delta) r^{t}+\left(\sigma^{2} / 2\right)-n\right]+\left(p^{2} \sigma^{2} / 2\right) .
$$

Hence

$$
\int_{0}^{T} \mathbb{E}\left[|k(t)|^{p}\right] d s=\frac{|k(0)|^{p}}{\overparen{\zeta}}[\exp (\widehat{\zeta} T)-1]
$$


Therefore,

$$
\begin{aligned}
& \mathbb{E}\left[\sup _{0 \leq t \leq T}\left|c^{*}(t)-c^{*}\right|^{p}\right] \\
\leq & 2|\rho \sigma|^{2 p} T^{3(p-2) / 2}\left(|\rho \sigma|^{p}+|\bar{\omega}|^{p}\right) \times \bar{\zeta}(p)\left(\left|c^{*}(0)-c^{*}\right|^{p}+\frac{|k(0)|^{p}}{\widehat{\zeta}}[\exp (\widehat{\zeta} T)-1]\right)
\end{aligned}
$$

Put

$C(p, T) \triangleq 2|\rho \sigma|^{2 p} T^{3(p-2) / 2}\left(|\rho \sigma|^{p}+|\bar{\omega}|^{p}\right) \bar{\zeta}(p)\left(\left|c^{*}(0)-c^{*}\right|^{p}+\frac{|k(0)|^{p}}{\widehat{\zeta}}[\exp (\widetilde{\zeta} T)-1]\right)$

Then we have

$$
\mathbb{E}\left[\sup _{0 \leq t \leq T}\left|c^{*}(t)-c^{*}\right|^{p}\right] \leq C(p, T)
$$

Thus, if $\rho \rightarrow 0$ or $\sigma \rightarrow 0$, then by Levi lemma we get

$$
\lim _{T \rightarrow \infty} \mathbb{E}\left[\sup _{0 \leq t \leq T}\left|c^{*}(t)-c^{*}\right|^{p}\right]=\mathbb{E}\left[\lim _{T \rightarrow \infty} \sup _{0 \leq t \leq T}\left|c^{*}(t)-c^{*}\right|^{p}\right] \rightarrow 0
$$

I

\section{Proof of Theorem 7}

By (26), we see that,

$$
\begin{aligned}
& d \Phi(t)=\left(\begin{array}{c}
-d k(t) \\
d y(t) \\
d c^{*}(t)
\end{array}\right) \\
& =\left(\begin{array}{c}
-\left[r^{s} A-\delta-(A-\delta) r^{t}-n+\sigma^{2}\right] \\
A\left[r^{s} A-\delta-(A-\delta) r^{t}-n+\sigma^{2}\right] \\
\rho\left[r^{s} A-\delta-(A-\delta) r^{t}-n+\sigma^{2}\right]
\end{array}\right) k(t) d t+\left(\begin{array}{c}
1 \\
-A \\
-\rho
\end{array}\right) \sigma k(t) d B(t) \\
& \triangleq\left(\begin{array}{lll}
\bar{\varsigma}_{1} & \bar{\varsigma}_{2} & \bar{\varsigma}_{3}
\end{array}\right)^{T} k(t) d t+\left(\begin{array}{lll}
1 & -A & -\rho
\end{array}\right)^{T} \sigma k(t) d B(t) \\
& \triangleq \vec{\zeta} k(t) d t+(1-A-\rho)^{T} \sigma k(t) d B(t) \\
& \triangleq \vec{f}(k(t)) d t+\vec{g}(k(t)) d B(t)
\end{aligned}
$$

Now, by Itô formula,

$$
\begin{aligned}
\left\|\Phi(t)-\Phi^{*}\right\|_{2}^{2} & =\left\|\Phi(0)-\Phi^{*}\right\|_{2}^{2}+2 \int_{0}^{t}\left\langle\Phi(s)-\Phi^{*}, \vec{f}(k(s))\right\rangle d s \\
& +2 \int_{0}^{t}\left\langle\Phi(s)-\Phi^{*}, \vec{g}(k(s)) d B(s)\right\rangle+\int_{0}^{t}\|\vec{g}(k(s))\|_{2}^{2} d s
\end{aligned}
$$


where $\langle\cdot, \cdot\rangle$ denotes the standard inner product. For $t_{1} \in[0, T]$, and $\zeta=\zeta(p), \forall p \geq 2$ we get

$$
\begin{aligned}
\sup _{0 \leq t \leq t_{1}}\left\|\Phi(t)-\Phi^{*}\right\|_{p}^{p} & \leq \zeta\left\{\left\|\Phi(0)-\Phi^{*}\right\|_{p}^{p}+\left[\int_{0}^{t_{1}}\left(\left(1+\rho^{2}+A^{2}\right) \sigma^{2}|k(s)|^{2}\right) d s\right]^{p / 2}\right. \\
& +\sup _{0 \leq t \leq t_{1}}\left|\int_{0}^{t}\left\langle\Phi(s)-\Phi^{*}, \vec{f}(k(s))\right\rangle d s\right|^{p / 2} \\
& \left.+\sup _{0 \leq t \leq t_{1}}\left|\int_{0}^{t}\left\langle\Phi(s)-\Phi^{*}, \vec{g}(k(s)) d B(s)\right\rangle\right|^{p / 2}\right\}
\end{aligned}
$$

It follows from Cauchy-Schwarz inequality that

$$
\begin{aligned}
& \sup _{0 \leq t \leq t_{1}}\left\|\Phi(t)-\Phi^{*}\right\|_{p}^{p} \\
\leq & \zeta\left\{\left\|\Phi(0)-\Phi^{*}\right\|_{p}^{p}+\left(1+\rho^{2}+A^{2}\right)^{p / 2}|\sigma|^{p} T^{(p-2) / 2} \int_{0}^{t_{1}}|k(s)|^{p} d s\right. \\
+ & \sup _{0 \leq t \leq t_{1}}\left|\int_{0}^{t}\left\langle\Phi(s)-\Phi^{*}, \vec{f}(k(s))\right\rangle d s\right|^{p / 2} \\
+ & \left.\sup _{0 \leq t \leq t_{1}}\left|\int_{0}^{t}\left\langle\Phi(s)-\Phi^{*}, \vec{g}(k(s)) d B(s)\right\rangle\right|^{p / 2}\right\}
\end{aligned}
$$

Taking expectations and for some $\underline{\zeta}=\underline{\zeta}(p)$, we have

$$
\begin{aligned}
& \mathbb{E}\left[\sup _{0 \leq t \leq t_{1}}\left\|\Phi(t)-\Phi^{*}\right\|_{p}^{p}\right] \\
\leq & \left(1+\rho^{2}+A^{2}\right)^{p / 2}|\sigma|^{p} T^{(p-2) / 2} \underline{\zeta}\left\{\mathbb{E}\left\|\Phi(0)-\Phi^{*}\right\|_{p}^{p}+\mathbb{E}\left[\int_{0}^{t_{1}}|k(s)|^{p} d s\right]\right. \\
& +\mathbb{E}\left[\sup _{0 \leq t \leq t_{1}}\left|\int_{0}^{t}\left\langle\Phi(s)-\Phi^{*}, \vec{f}(k(s))\right\rangle d s\right|^{p / 2}\right] \\
& \left.+\mathbb{E}\left[\sup _{0 \leq t \leq t_{1}}\left|\int_{0}^{t}\left\langle\Phi(s)-\Phi^{*}, \vec{g}(k(s)) d B(s)\right\rangle\right|^{p / 2}\right]\right\}
\end{aligned}
$$


Applying Burkholder-Davis-Gundy inequality, for some $\tilde{\zeta}=\tilde{\zeta}(p)$,

$$
\begin{aligned}
& \mathbb{E}\left[\sup _{0 \leq t \leq t_{1}}\left\|\Phi(t)-\Phi^{*}\right\|_{p}^{p}\right] \\
\leq & \left(1+\rho^{2}+A^{2}\right)^{p / 2}|\sigma|^{p} T^{(p-2) / 2} \tilde{\zeta}\left\{\mathbb{E}\left\|\Phi(0)-\Phi^{*}\right\|_{p}^{p}\right. \\
+ & \int_{0}^{t_{1}} \mathbb{E}|k(s)|^{p} d s+\mathbb{E}\left[\int_{0}^{t_{1}}\left\|\Phi(t)-\Phi^{*}\right\|_{2}^{2}\|\vec{f}(k(s))\|_{2}^{2} d s\right]^{p / 4} \\
+ & \left.\mathbb{E}\left[\int_{0}^{t_{1}}\left\|\Phi(t)-\Phi^{*}\right\|_{2}^{2}\|\vec{g}(k(s))\|_{2}^{2} d s\right]^{p / 4}\right\}
\end{aligned}
$$

Next, by the Young inequality (see, Higham et al, 2003) and Hölder inequality,

$$
\begin{aligned}
& \mathbb{E}\left[\int_{0}^{t_{1}}\left\|\Phi(t)-\Phi^{*}\right\|_{2}^{2}\|\vec{f}(k(s))\|_{2}^{2} d s\right]^{p / 4} \\
\leq & \mathbb{E}\left[\sup _{0 \leq s \leq t_{1}}\left\|\Phi(t)-\Phi^{*}\right\|_{2}^{p / 2}\left(\int_{0}^{t_{1}}\|\vec{f}(k(s))\|_{2}^{2} d s\right)^{p / 4}\right] \\
\leq & \frac{1}{2\left(2\left(1+\rho^{2}+A^{2}\right)^{p / 2}|\sigma|^{p} T^{(p-2) / 2} \tilde{\zeta}\right)} \mathbb{E}\left[\sup _{0 \leq t \leq t_{1}}\left\|\Phi(t)-\Phi^{*}\right\|_{p}^{p}\right] \\
& +\frac{\left(2\left(1+\rho^{2}+A^{2}\right)^{p / 2}|\sigma|^{p} T^{(p-2) / 2} \tilde{\zeta}\right)}{2} \mathbb{E}\left[\int_{0}^{t_{1}}\|\vec{f}(k(s))\|_{2}^{2} d s\right]^{p / 2} \\
\leq & \frac{1}{2\left(2\left(1+\rho^{2}+A^{2}\right)^{p / 2}|\sigma|^{p} T^{(p-2) / 2} \tilde{\zeta}\right)} \mathbb{E}\left[\sup _{0 \leq t \leq t_{1}}\left\|\Phi(t)-\Phi^{*}\right\|_{p}^{p}\right] \\
& +\frac{\left(2\left(1+\rho^{2}+A^{2}\right)^{p / 2}|\sigma|^{p} T^{(p-2) / 2} \tilde{\zeta}\right)}{2} T^{(p-2) / 2}\|\vec{\varsigma}\|_{2}^{p} \mathbb{E}\left[\int_{0}^{t_{1}}|k(s)|^{p} d s\right]
\end{aligned}
$$

Similarly, we get

$$
\begin{aligned}
& \mathbb{E}\left[\int_{0}^{t_{1}}\left\|\Phi(t)-\Phi^{*}\right\|_{2}^{2}\|\vec{g}(k(s))\|_{2}^{2} d s\right]^{p / 4} \\
\leq & \frac{1}{2\left(2\left(1+\rho^{2}+A^{2}\right)^{p / 2}|\sigma|^{p} T^{(p-2) / 2} \tilde{\zeta}\right)} \mathbb{E}\left[\sup _{0 \leq t \leq t_{1}}\left\|\Phi(t)-\Phi^{*}\right\|_{p}^{p}\right] \\
+ & \frac{\left(2\left(1+\rho^{2}+A^{2}\right)^{p / 2}|\sigma|^{p} T^{(p-2) / 2} \tilde{\zeta}\right)}{2} T^{(p-2) / 2}\left(1+\rho^{2}+A^{2}\right)^{p / 2}|\sigma|^{p} \mathbb{E}\left[\int_{0}^{t_{1}}|k(s)|^{p} d s\right]
\end{aligned}
$$


Hence, substituting (H.2) and (H.3) into (H.1) yields,

$$
\begin{aligned}
& \mathbb{E}\left[\sup _{0 \leq t \leq t_{1}}\left\|\Phi(t)-\Phi^{*}\right\|_{p}^{p}\right] \leq 2\left(1+\rho^{2}+A^{2}\right)^{p / 2}|\sigma|^{p} T^{(p-2) / 2} \tilde{\zeta}\left\{\mathbb{E}\left\|\Phi(0)-\Phi^{*}\right\|_{p}^{p}\right. \\
+ & \int_{0}^{t_{1}} \mathbb{E}|k(s)|^{p} d s+\left(1+\rho^{2}+A^{2}\right)^{p / 2}|\sigma|^{p} \tilde{\zeta} T^{p-2} \\
\times & \left.\mathbb{E}\left[\int_{0}^{t_{1}}|k(s)|^{p} d s\right]\left(\|\vec{\zeta}\|_{2}^{p}+\left(1+\rho^{2}+A^{2}\right)^{p / 2}|\sigma|^{p}\right)\right\}
\end{aligned}
$$

Then there exists some $\bar{\zeta}=\bar{\zeta}(p)$ such that,

$$
\begin{aligned}
& \mathbb{E}\left[\sup _{0 \leq t \leq t_{1}}\left\|\Phi(t)-\Phi^{*}\right\|_{p}^{p}\right] \\
\leq & 2\left(1+\rho^{2}+A^{2}\right)^{p}|\sigma|^{2 p} T^{3(p-2) / 2}\left(\|\vec{\varsigma}\|_{2}^{p}+\left(1+\rho^{2}+A^{2}\right)^{p / 2}|\sigma|^{p}\right) \bar{\zeta} \\
\times & \left(\mathbb{E}\left[\left\|\Phi(0)-\Phi^{*}\right\|_{p}^{p}\right]+\int_{0}^{t_{1}} \mathbb{E}|k(s)|^{p} d s\right) \\
\leq & 2\left(1+\rho^{2}+A^{2}\right)^{p}|\sigma|^{2 p} T^{3(p-2) / 2}\left(\|\vec{\varsigma}\|_{2}^{p}+\left(1+\rho^{2}+A^{2}\right)^{p / 2}|\sigma|^{p}\right) \bar{\zeta}(p) \\
\times & \left(\mathbb{E}\left[\left\|\Phi(0)-\Phi^{*}\right\|_{p}^{p}\right]+\int_{0}^{T} \mathbb{E}|k(s)|^{p} d s\right)
\end{aligned}
$$

Since by (6),

$$
k(t)=k(0) \exp \left\{\left[r^{s} A-\delta-(A-\delta) r^{t}+\left(\sigma^{2} / 2\right)-n\right] t-\sigma B(t)\right\}
$$

Thus

$$
\begin{aligned}
\mathbb{E}\left[|k(t)|^{p}\right] & =|k(0)|^{p} \mathbb{E}\left[\exp \left\{p\left[r^{s} A-\delta-(A-\delta) r^{t}+\left(\sigma^{2} / 2\right)-n\right] t-p \sigma B(t)\right\}\right] \\
& =|k(0)|^{p} \exp (\widehat{\zeta}(p) t)
\end{aligned}
$$

where

$$
\widehat{\zeta}(p) \triangleq p\left[r^{s} A-\delta-(A-\delta) r^{t}+\left(\sigma^{2} / 2\right)-n\right]+\left(p^{2} \sigma^{2} / 2\right)
$$

Hence

$$
\int_{0}^{T} \mathbb{E}\left[|k(t)|^{p}\right] d s=\frac{|k(0)|^{p}}{\widehat{\zeta}}[\exp (\widehat{\zeta} T)-1]
$$


Therefore,

$$
\begin{aligned}
& \mathbb{E}\left[\sup _{0 \leq t \leq T}\left\|\Phi(t)-\Phi^{*}\right\|_{p}^{p}\right] \\
\leq & 2\left(1+\rho^{2}+A^{2}\right)^{p}|\sigma|^{2 p} T^{3(p-2) / 2}\left(\|\vec{\zeta}\|_{2}^{p}+\left(1+\rho^{2}+A^{2}\right)^{p / 2}|\sigma|^{p}\right) \\
& \times \bar{\zeta}(p)\left(\left\|\Phi(0)-\Phi^{*}\right\|_{p}^{p}+\frac{|k(0)|^{p}}{\overparen{\zeta}}[\exp (\widehat{\zeta} T)-1]\right)
\end{aligned}
$$

Put

$$
\begin{aligned}
C(p, T) & \triangleq 2\left(1+\rho^{2}+A^{2}\right)^{p}|\sigma|^{2 p} T^{3(p-2) / 2}\left(\|\vec{\zeta}\|_{2}^{p}+\left(1+\rho^{2}+A^{2}\right)^{p / 2}|\sigma|^{p}\right) \\
& \times \bar{\zeta}(p)\left(\left\|\Phi(0)-\Phi^{*}\right\|_{p}^{p}+\frac{|k(0)|^{p}}{\widehat{\zeta}}[\exp (\widehat{\zeta} T)-1]\right)
\end{aligned}
$$

Then we have

$$
\mathbb{E}\left[\sup _{0 \leq t \leq T}\left\|\Phi(t)-\Phi^{*}\right\|_{p}^{p}\right] \leq C(p, T)
$$

Thus, if $\sigma \rightarrow 0$, by Levi lemma we get the following desired result,

$$
\lim _{T \rightarrow \infty} \mathbb{E}\left[\sup _{0 \leq t \leq T}\left\|\Phi(t)-\Phi^{*}\right\|_{p}^{p}\right]=\mathbb{E}\left[\lim _{T \rightarrow \infty} \sup _{0 \leq t \leq T}\left\|\Phi(t)-\Phi^{*}\right\|_{p}^{p}\right] \rightarrow 0
$$

\section{REFERENCES}

Aghion, P., 2004. Growth and Development: A Schumpeterian Approach. Annals of Economics and Finance 5, 1-25.

Araujo, A. and J.A. Scheinkman, 1977. Smoothness, Comparative Dynamics, and the Turnpike Property. Econometrica 45, 601-620.

Barro, R.J., 1990. Government Spending in a Simple Model of Endogenous Growth. Journal of Political Economy 98, 103-125.

Bewley, T., 1982. An Integration of Equilibrium Theory and Turnpike Theory. Journal of Mathematical Economics 10, 233-267.

Bomze, I.M., 1991. Cross entropy minimization in uninvadable states of complex populations. Journal of Mathematical Biology 30, 73-87.

Coles, J.L., 1985. Equilibrium Turnpike Theory with Constant Returns to Scale and Possibly Heterogeneous Discount Factors. International Economic Review 26, 671679 .

Drandakis, E.M., 1966. On Efficient Accumulation Paths in the Closed Production Model. Econometrica 34, 331-346. 
Gale, D., 1967. On Optimal Development in a Multi-Sector Economy. Review of Economic Studies 34, 1-18.

Gantz, D.T., 1980. A Strong Turnpike Theorem for a Nonstationary von NeumannGale Production Model. Econometrica 48, 1777-1790.

Gong, L. and H. Zou, 2000. Foreign Aid Reduces Domestic Capital Accumulation and Increases Foreign Borrowing: A Theoretical Analysis. Annals of Economics and Finance 1, 147-163.

Gong, L. and H. Zou, 2002. Fiscal Federalism, Public Capital Formation, and Endogenous Growth. Annals of Economics and Finance 4, 471-490.

Higham, D.J., X.R. Mao and A.M. Stuart, 2003. Strong Convergence of Euler-Type Methods for Nonlinear Stochastic Differential Equations. SIAM Journal on Numerical Analysis 40, 1041-1063.

Joshi, S., 1997. Turnpike Theorem in Nonconvex and Nonstationary Environment. International Economic Review 38, 225-248.

Karatzas, I. and S. E. Shreve, 1991. Brownian Motion and Stochastic Calculus. Volume 113 of Graduate Texts in Mathematics. New York: Springer-Verlag, second edition.

Kurz, M., 1965. Optimal Paths of Capital Accumulation Under the Minimum Time Objective. Econometrica 33, 42-66.

Imhof, L.A., 2005. The Long-Run Behavior of the Stochastic Replicator Dynamics. Annals of Applied Probability 15, 1019-1045.

McKenzie, L., 1963. Turnpike Theorems for a Generalized Leontief Model. Econometrica 31, 165-180

McKenzie, L., 1976. Turnpike Theory. Econometrica 44, 841-865.

McKenzie, L., 1982. A Primal Route to the Turnpike and Liapounov Stability. Journal of Economic Theory 27, 194-209.

Merton, R.C., 1975. An Asymptotic Theory of Growth Under Uncertainty. Review of Economic Studies 42, 375-393.

Morishima, M., 1961. Proof of a Turnpike Theorem: The "No Joint Production" Case. Review of Economic Studies 28, 89-97.

Morishima, M., 1965. On the Two Theorems of Growth Economics: A Mathematical Exercise. Econometrica 33, 829-840.

Øksendal, B., 2003. Stochastic Differential Equations. Universitext. Berlin: SpringerVerlag, sixth edition.

Øksendal, B. and A.Sulem, 2007. Applied Stochastic Control of Jump Diffusions. Berlin: Springer- Verlag, second edition.

Radner, R., 1961. Paths of Economic Growth that are Optimal with Regard only to Final States: A Turnpike Theorem. Review of Economic Studies 28, 98-104.

Rebelo, S., 1991. Long-run Policy Analysis and Long-run Growth. Journal of Political Economy 99, 500-521.

Tsukui, J., 1966. Turnpike Theorem in a Generalized Dynamic Input-Output System. Econometrica 34, 396-407.

Tsukui, J., 1967. The Consumption and the Output Turnpike Theorems in a von Neumann Type of Model — Finite Term Problem. Review of Economic Studies 34, 85-93. 
Turnovsky, S.J., 2000. Fiscal Policy, Elastic Labor Supply, and Endogenous Growth. Journal of Monetary Economics 45, 185-210.

Winter, S.G., Jr., 1967. The Norms of a Closed Technology and the Straight-Downthe-Turnpike Theorem. Review of Economic Studies 34, 67-84.

Yano, M., 1984. The Turnpike of Dynamic General Equilibrium Paths and Its Insensitivity to Initial Conditions. Journal of Mathematical Economics 13, 235-254.

Yano, M., 1984. Competitive Equilibria on Turnpikes in a McKenzie Economy, I: A Neighborhood Turnpike Theorem. International Economic Review 25, 695-717.

Yano, M., 1985. Competitive Equilibria on Turnpikes in a McKenzie Economy, II: An Asymptotic Turnpike Theorem. International Economic Review 26, 661-669.

Yano, M., 1998. On the Dual Stability of a Von Neumann Facet and the Inefficacy of Temporary Fiscal Policy. Econometrica 66, 427-451. 